\title{
ARTICLE PARylation regulates stress granule dynamics, phase separation, and neurotoxicity of disease-related RNA-binding proteins
}

Yongjia Duan ${ }^{1,2}$, Aiying $\mathrm{Du}^{1}$, Jinge $\mathrm{Gu}^{1,2}$, Gang Duan ${ }^{1}$, Chen Wang ${ }^{1,2}$, Xinrui Gui ${ }^{1,2}$, Zhiwei Ma ${ }^{1,3}$, Beituo Qian ${ }^{1,2}$, Xue Deng ${ }^{1,2}$, Kai Zhang ${ }^{1,2}$, Le Sun ${ }^{1,2}$, Kuili Tian ${ }^{1}$, Yaoyang Zhang ${ }^{1,2}$, Hong Jiang ${ }^{1,2}$, Cong Liu (iD) ${ }^{1,2}$ and Yanshan Fang (iD)

Mutations in RNA-binding proteins (RBPs) localized in ribonucleoprotein (RNP) granules, such as hnRNP A1 and TDP-43, promote aberrant protein aggregation, which is a pathological hallmark of various neurodegenerative diseases, such as amyotrophic lateral sclerosis (ALS) and frontotemporal dementia (FTD). Protein posttranslational modifications (PTMs) are known to regulate RNP granules. In this study, we investigate the function of poly(ADP-ribosyl)ation (PARylation), an important PTM involved in DNA damage repair and cell death, in RNP granule-related neurodegeneration. We reveal that PARylation levels are a major regulator of the assembly-disassembly dynamics of RNP granules containing disease-related RBPs, hnRNP A1 and TDP-43. We find that hnRNP A1 can both be PARylated and bind to PARylated proteins or poly(ADP-ribose) (PAR). We further uncover that PARylation of hnRNP A1 at K298 controls its nucleocytoplasmic transport, whereas PAR-binding via the PAR-binding motif (PBM) of hnRNP A1 regulates its association with stress granules. Moreover, we reveal that PAR not only dramatically enhances the liquid-liquid phase separation of hnRNP A1, but also promotes the co-phase separation of hnRNP A1 and TDP-43 in vitro and their interaction in vivo. Finally, both genetic and pharmacological inhibition of PARP mitigates hnRNP A1- and TDP-43-mediated neurotoxicity in cell and Drosophila models of ALS. Together, our findings suggest a novel and crucial role for PARylation in regulating the dynamics of RNP granules, and that dysregulation in PARylation and PAR levels may contribute to ALS disease pathogenesis by promoting protein aggregation.

Cell Research (2019) 29:233-247; https://doi.org/10.1038/s41422-019-0141-z

\section{INTRODUCTION}

Eukaryotic genomes encode a large number of RBPs that can associate with RNAs to form ribonucleoprotein (RNP) complexes. These RBPs contain conserved RNA binding domain(s) and protein-protein interaction domain(s). They are present in both nucleus and cytoplasm, where they play a major role in RNA homeostasis including RNA processing, transport and turnover. ${ }^{1-3}$ RBPs can form granules by liquid-liquid phase separation (LLPS), and aberrant RNP granules enriched of irreversible amyloid aggregations may promote the pathogenesis of human neurodegenerative diseases, including amyotrophic lateral sclerosis (ALS) and frontotemporal dementia (FTD). ${ }^{4,5}$ Posttranslational modifications (PTMs) such as phosphorylation, ubiquitination and acetylation are known to regulate the assembly and function of RNP granules. ${ }^{6-9}$ Recently, methylation and phosphorylation were reported to modulate phase transition of hnRNP A2 and FUS. ${ }^{10-13}$ However, the function of other important PTMs in regulating LLPS and RNP granules remains to be explored.

Poly(ADP-ribosyl)ation (PARylation) is a reversible PTM process by which poly(ADP-ribose) (PAR) polymerases (PARPs) add ADP- ribose (ADPr) units to the Glu, Asp, Lys, Arg or Ser residue of a protein, ${ }^{14,15}$ whereas enzymes such as PAR glycohydrolase (PARG) remove them. ${ }^{16,17}$ The opposing effects of PARPs and PARG in regulating protein PARylation play an important role in a variety of cellular functions, including chromatin remodeling, DNA repair, transcription regulation and cell death. ${ }^{18-21}$ Dysregulation in PARylation is therefore involved in various disease conditions such as cancer, neurodegeneration, oxidative stress, neural injury, and regeneration. ${ }^{22-25}$ Interestingly, PAR as well as some PARPs and PARG are found in cytoplasmic stress granules (SGs) and may regulate microRNA-mediated translational repression and mRNA cleavage. $^{26}$

Familial ALS-associated mutations in the genes encoding several RBPs, such as the heterogeneous nuclear ribonucleoprotein $\mathrm{A} 1$ (hnRNP $\mathrm{A} 1$, encoded by HNRNPA1) and the TAR DNA binding protein $43 \mathrm{kDa}$ (TDP-43, encoded by TARDBP), are known to promote protein aggregation. ${ }^{27,28}$ hnRNP A1 and TDP-43 are predominantly nuclear proteins that can shuttle between the nucleus and cytoplasm. In the nucleus, they can interact with each other and regulate multiple aspects of RNA processing and metabolism. Upon stress, they are translocated to the cytoplasm

\footnotetext{
${ }^{1}$ Interdisciplinary Research Center on Biology and Chemistry, Shanghai Institute of Organic Chemistry, Chinese Academy of Sciences, Shanghai 201210 , China and ${ }^{2}$ University of Chinese Academy of Sciences, Beijing 100049, China

Correspondence: Cong Liu (liulab@sioc.ac.cn) or Yanshan Fang (fangys@sioc.ac.cn)

${ }^{3}$ Present address: Shanghai Institutes for Biological Sciences, Chinese Academy of Sciences, Shanghai 200031, China

These authors contributed equally: Yongjia Duan, Aiying Du, Jinge Gu.
}

Received: 28 August 2018 Accepted: 7 January 2019

Published online: 6 February 2019 
along with a variety of other RBPs, forming SGs and sequestering non-translating mRNAs. ${ }^{29-32}$ In this study, we show that PARylation regulates SG dynamics, phase separation, protein-protein interaction, and the neurotoxicity of hnRNP A1 and TDP-43. Given that both the RNAi knockdown of PARP1 and the application of a PARP inhibitor significantly suppress the hnRNP A1 and TDP-43mediated neurodegeneration, PARylation is functionally important that implicates potential therapeutic values of PARP inhibitors for the treatment of ALS and other related diseases.

\section{RESULTS}

PARylation regulates the dynamics of the SGs containing ALSrelated $\mathrm{RBPs}$

Although PARylation occurs primarily on PARP proteins, the association of PAR with SGs and ALS-related RBPs has been observed $^{26,33,34}$ (Supplementary information, Fig. S1). To determine whether PARylation regulates the SGs containing diseaserelated RBPs such as TDP-43 and hnRNP A1, we tested the effect of Olaparib, a Food and Drug Administration (FDA)-approved PARP inhibitor for cancer treatment, ${ }^{35}$ on the SG recruitment of TDP-43 and hnRNP A1 when cells were stressed (arsenite, $100 \mu \mathrm{M}$ ). SGs were examined by immunocytochemistry using a SG marker Tcell-restricted intracellular antigen 1 (TIA-1)-related protein (TIAR). ${ }^{36}$ In response to the arsenic stress, TDP-43 and hnRNP A1 were recruited to SGs. In contrast, inhibition of PARP by Olaparib significantly delayed the assembly of SGs as well as the recruitment of TDP-43 (Fig. 1a-c) and hnRNP A1 (Supplementary information, Fig. S2) to SGs. Further, we downregulated the expression levels of the PAR hydrolysis enzyme PARG with the small interfering RNA (siRNA) to increase the PARylation levels in the cell. SGs were induced by arsenite treatment for $30 \mathrm{~min}$, followed by a washout experiment to determine the kinetics of the SG disassembly (Fig. 1e). In addition to delaying disassembly as previously reported, ${ }^{26}$ si-PARG also drastically delayed the retrieval of TDP-43 (Supplementary information, Fig. S3) and hnRNP A1 (Fig. 1d-f) from SGs. Thus, the PARylation level was identified to be a major factor regulating the assemblydisassembly dynamics of SGs associated with ALS-related RNPs.

PARylation and PAR-binding of hnRNP A1

Since PARylation levels affected the dynamics of RNP granules containing TDP-43 and hnRNP A1, we then examined whether these proteins were PARylated or associated with other PARylated proteins in cells. The HA-tagged TDP-43 or Flag-tagged hnRNP A1 was expressed and immunoprecipitated from HeLa cells with the anti-HA or anti-Flag antibodies. They were then evaluated by Western blotting with a pan-PAR antibody (anti-PAR) that recognizes both the mono-ADPr and poly-ADPr. No basal PARylation of TDP-43 was detected in the cells (Fig. 2a). To boost PARP1 activity, we treated the cells with $\mathrm{H}_{2} \mathrm{O}_{2}(500 \mu \mathrm{M}, 10 \mathrm{~min})$, which could induce PARP1 activation in cells. ${ }^{14}$ With activated PARP1, the PARylation levels of hnRNP A1 were markedly increased (Fig. 2b), however, still no PARylation of TDP-43 was detected (Fig. 2a). Nevertheless, this result does not exclude the possibility that PARylation of TDP-43 occurs at a level below the detection sensitivity of our assay or that TDP-43 can bind to free PAR polymers in cells. In contrast, the hnRNP A1 immunoprecipitated from HeLa cells showed a steady-state level of PARylation as well as an $\mathrm{H}_{2} \mathrm{O}_{2}$-induced elevation of PARylation. In addition, there were other PARylated proteins coimmunoprecipitated with hnRNP A1, especially after treatment with $\mathrm{H}_{2} \mathrm{O}_{2}$ (bands indicated by "\#" in Fig. 2b). Treating the immunoprecipitates with PARG drastically reduced the intensity of the hnRNP A1 PARylation bands as well as the proteins co-immunoprecipitated with hnRNP A1 in the anti-PAR blot (Fig. 2c), confirming these bands to be indeed a result of PARylation.

Next, we assessed whether these proteins could be PARylated in vitro. The full-length TDP-43 (TDP-43-FL) protein expressed in $E$. coli was extremely insoluble. We tried several different expression vectors with different purification tags and induction temperatures, but failed to produce sufficient amount of soluble TDP-43-FL protein for the use in the in vitro assays (Supplementary information, Fig. S4a-e). Therefore, an alternative approach was taken, which involved the expression of TDP-43 in two truncations, namely TDP-43 ${ }^{1-274}$ and TDP-43 $3^{274-414}$ (Supplementary information, Fig. S4a, f-g). Purified TDP-43 truncations as well as full-length hnRNP A1 protein were subjected to an in vitro PARylation reaction. Single-strand DNA (ssDNA) was added to activate PARP1 in the in vitro system and the PARylation levels were examined by SDS-PAGE and immunoblotting with the anti-PAR antibody. ssDNA mimics DNA single-strand breaks, the most common form of DNA damage in cells, and can induce PARP1 activation in in vitro PARylation assays. ${ }^{37,38}$ Indeed, the activation of PARP1 by ssDNA was evident through the PARylation of PARP1 itself (the smears above $115 \mathrm{kDa}$ in Fig. 2d). With activated PARP1, the PARylation bands of TDP-43 ${ }^{1-274}$ and hnRNP A1 showed increased intensity as well as up-shifting smears, whereas no significant induction of TDP-43 $3^{274-414}$ PARylation was observed (Fig. 2d). Note that, due to the heterogeneity in the length of the poly-ADPr polymer attached (Fig. 2e), the PAR immunoreactivity did not necessarily correspond to the protein abundance or manifest a massive mobility shift of the total protein in the Coomassie staining (Fig. 2d).

hnRNP A1 contains a PARylation site at K298 and a PAR-binding motif (PBM)

The human hnRNP A1 protein contains two closely-related RNA recognition motifs (RRMs) in the $\mathrm{N}$-terminal region and a low complexity (LC), glycine-rich domain (GRD) in the C-terminal region that includes an RGG box RNA binding domain and a M9 nuclear targeting sequence ${ }^{39}$ (Fig. 2f). In addition, previous mass spectrometry-based studies suggested that hnRNP A1 might contain a putative PARylation site at K298 and a PAR-binding motif (PBM) between the two RRM domains at amino acid (aa) 92-113. ${ }^{14,34}$ To validate and characterize the PARylation site and the $\mathrm{PBM}$ region, the constructs were generated to express the Flag-tagged hnRNP A1 of the PARylation site mutant (K298A) or the PBM mutant (R92A-K105/106 V, referred to as PBM $^{\text {mut }}$ ) in the cells (Fig. 2f). To examine the impact of PARylation and PARbinding on hnRNP A1, we transfected cells with Flag-tagged wildtype (WT), K298A or PBM ${ }^{\text {mut }}$ hnRNP A1 and then treated with $\mathrm{H}_{2} \mathrm{O}_{2}$. The cell lysates were examined by immunoprecipitation (IP) with the anti-Flag and Western blotting with the anti-PAR. Compared to WT hnRNP A1, the K298A mutant showed markedly reduced PARylation but a similar level of co-immunoprecipitation (co-IP) of other PARylated proteins as that of the WT hnRNP A1 (Fig. $2 \mathrm{~g}$ ). In contrast, the $\mathrm{PBM}^{\mathrm{mut}}$ showed a striking reduction of the co-immunoprecipitation of other PARylated proteins, whereas its own PARylation was not reduced but unexpectedly increased (Fig. 2g). The loss of PAR-binding capacity of PBM ${ }^{\text {mut }}$ was further confirmed using an in vitro dot-blot binding assay (Fig. $2 \mathrm{~h}$, i). Of note, PBM $^{\text {mut }}$ showed an up-shifted PARylation smear (Fig. $2 \mathrm{~g}$ ) to a similar extent as that of hnRNP A1 in the in vitro PARylation assay (Fig. 2d), indicating that the hnRNP A1 protein is capable of being massively PARylated when induced. In addition, these data suggest that binding to PAR and/or PARylated proteins via the PBM may prevent hyper-PARylation of hnRNP A1 at K298.

PARylation and PAR-binding are differentially required for cytoplasmic translocation and SG association of hnRNP A1 We showed that the cellular PARylation levels affected the recruitment and recovery of hnRNP A1 to and from SGs (Fig. 1d 1 f; Supplementary information, Fig. S2). However, it was unclear whether PARylation directly regulated the SG association of hnRNP A1 or indirectly by altering the overall SG dynamics. To address this question, we expressed the PARylation mutant K298A 
a

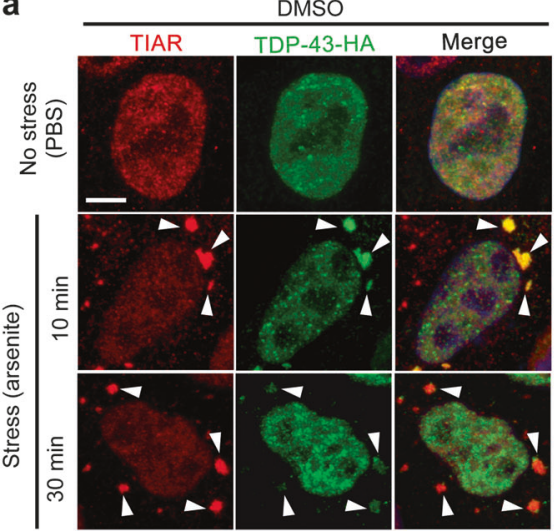

d

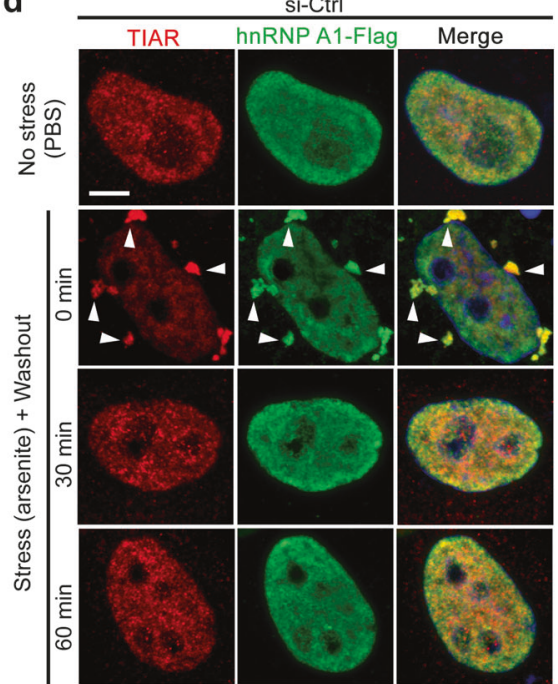

Olaparib

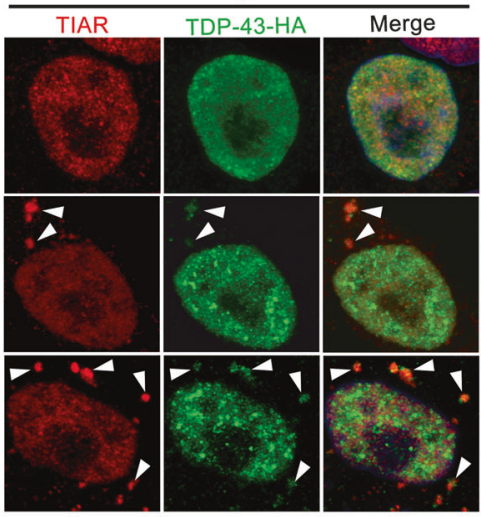

si-PARG

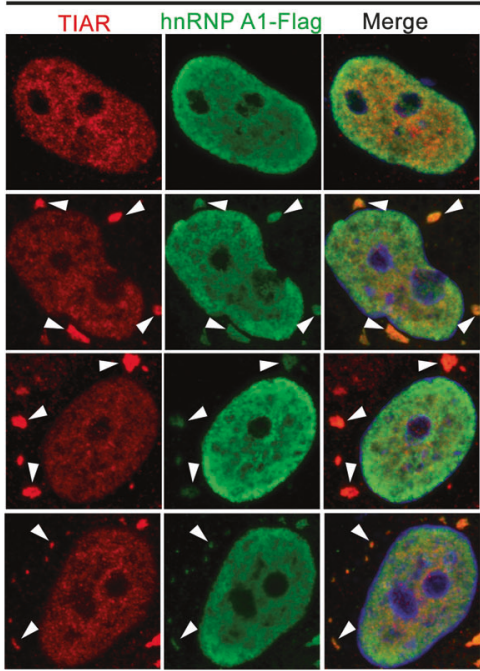

b
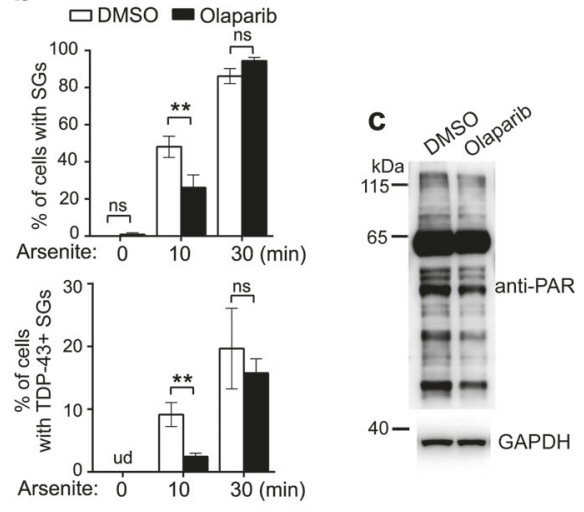

f
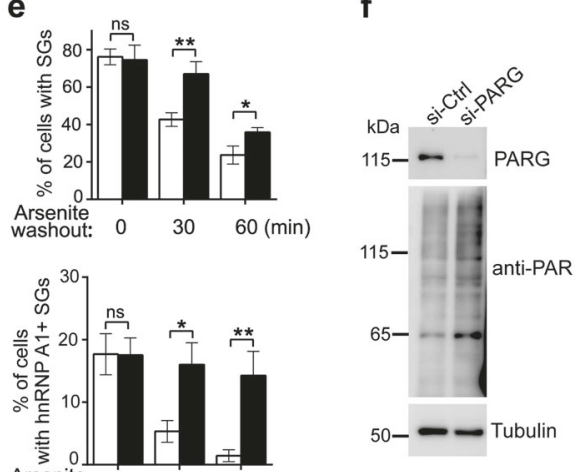

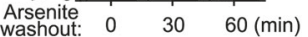

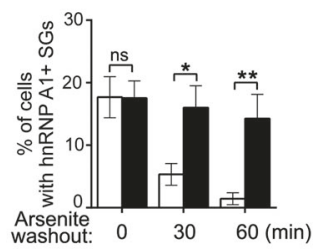

Fig. 1 The PARylation levels influence the assembly-disassembly dynamics of SGs containing ALS-related RNPs. a-c PARP inhibition suppresses SG assembly. a Representative confocal images of HeLa cells treated with PBS (no stress) or $100 \mu \mathrm{M}$ arsenite (stress) for the indicated time in the absence (DMSO) or presence of the PARP inhibitor Olaparib $(20 \mu \mathrm{M})$. All cells are transfected with TDP-43-HA (anti-HA) and stained for SGs by the anti-TIAR (arrowheads); the merged images with the DAPI staining of DNA (blue) are shown. b The percentage of cells with SGs and TDP-43 + SGs from a is quantified. c Western blot analysis confirms the decrease in the overall PARylation levels by Olaparib, with GAPDH as a loading control. $\mathbf{d}-\mathbf{f}$ Hydrolysis of PAR by PARG is required for SG disassembly. $\mathbf{d}$ Cells transfected with the siRNA (si-PARG) or the scrambled siRNA (si-Ctrl) are stressed with arsenite $(100 \mu \mathrm{M})$ for $30 \mathrm{~min}$ and then washed for the indicated time. All cells are transfected with the hnRNP A1-Flag (anti-Flag) and stained for SGs with the anti-TIAR (arrowheads); the merged images with the DAPI staining for DNA (blue) are shown. e Percentage of the cells with SGs or hnRNPA1 + SGs from (d); $\mathbf{f}$ Western blot analysis confirms PARG KD and the increase in the overall PARylation levels in the cells by si-PARG. Data are shown as the mean \pm SEM; $n=$ over 100 cells for each condition, pooled results of three independent repeats; ${ }^{*} p<0.05,{ }^{* *} p<0.01 ; \mathrm{ns}$, not significant; ud, undetected, Student's $t$-test. Scale bars $5 \mu \mathrm{m}$

and PAR-binding deficient PBM ${ }^{\text {mut }}$ of hnRNP A1 in HeLa cells and examined their cellular localization before and after stress. In the absence of stress, while WT and K298A of hnRNP A1 were predominantly nuclear, the $\mathrm{PBM}^{\text {mut }}$ also formed some cytoplasmic foci that did not co-localize with the SG marker TIAR (Fig. 3a). Of note, the PBM is not located within the RGG or M9 domain, the known nuclear localization sequences (NLS) of hnRNP A1.40,41 Hence, the PAR-binding at the PBM may be an additional mechanism regulating the nuclear import of hnRNP A1.

Next, we stressed the cells with arsenite $(100 \mu \mathrm{M}, 30 \mathrm{~min})$, which significantly induced the formation of SGs in cells expressing the WT, K298A or PBM ${ }^{\text {mut }}$ hnRNP A1 (Fig. 3a). Thus, the overall ability of the cells to form SGs was not affected by mutant hnRNP A1 (Fig. 3b). In response to stress, WT hnRNPA1 showed a significant cytoplasmic translocation and co-localized with TIAR-labeled SGs (Fig. 3a, C). Interestingly, the K298A mutant was not recruited into SGs and remained in the nucleus (Fig. 3a, c). Upon stress, some $\mathrm{PBM}^{\text {mut }}$ was recruited to TIAR-labeled SGs (Fig. 3a), but the induction was to a less extent than that of WT hnRNP A1 (Fig. 3c).
In addition, the percentage of cells with "PBM" ${ }^{\text {mut }}+/ \mathrm{TIAR}^{-}$" cytoplasmic foci showed no difference before and after stress (Fig. 3a, d-e), indicating a failure of the abnormal PBM ${ }^{\text {mut }}$ cytoplasmic foci to respond to stress. Together, these data suggest that PARylation of hnRNP A1 at K298A is required for its cytoplasmic translocation, whereas its binding to either PAR or PARylated proteins regulates its sorting and/or delivery to SGs.

The ALS-linked hnRNP A1 P288A mutation resides in the NLS and shows an accumulation of hnRNP A1 protein in cytoplasm and increased SG localization. ${ }^{42}$ We confirmed the effects of the P288A mutation in our system (Supplementary information, Fig. S5). Interestingly, P288A/K298A double mutant behaved similarly to that of P288A, exhibiting cytoplasmic accumulation co-localized with SGs, which was further increased upon stress (Supplementary information, Fig. S5a-c). The result that the P288A/K298A double mutant did not show any defect in SG association once it was localized to the cytoplasm confirms that PARylation at K298 is not required for SG localization. Moreover, the data suggest that disease-associated P288A mutation might 


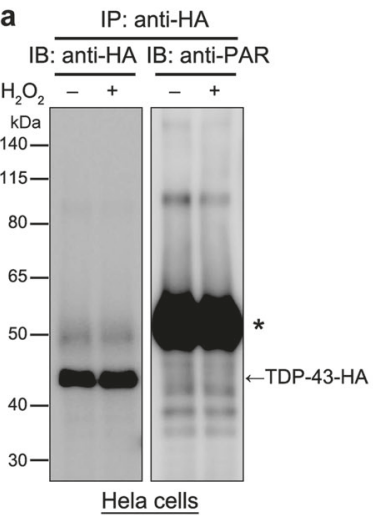

b

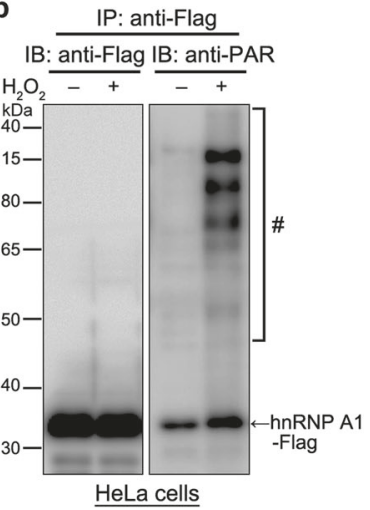

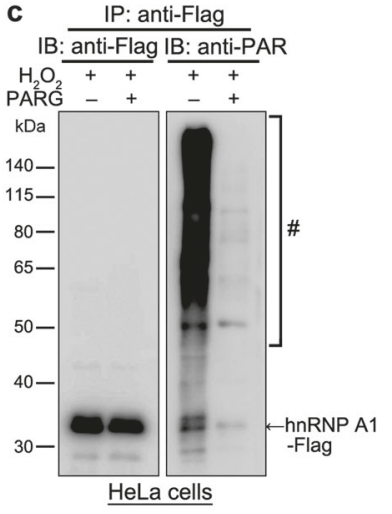

g

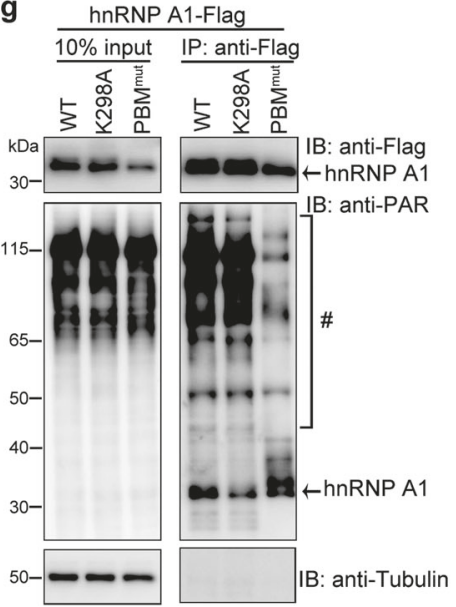

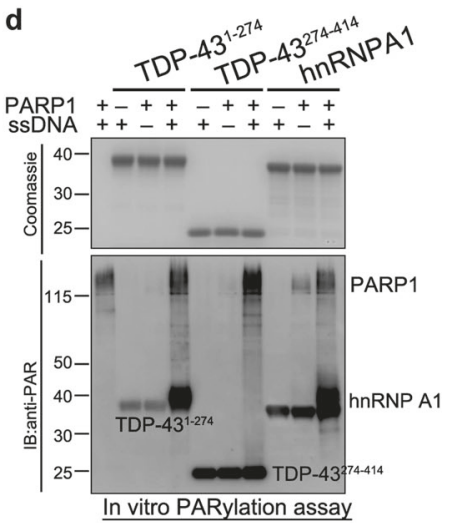

h
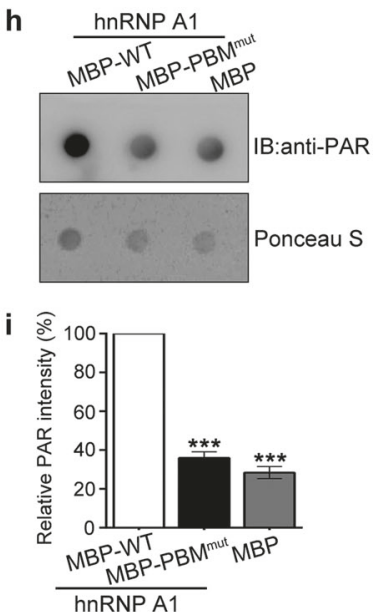

Fig. 2 PARylation and PAR binding of TDP-43 and hnRNP A1 in vitro and in cells. a, b HA-tagged TDP-43 (a) or Flag-tagged hnRNP A1 (b) is expressed and immunoprecipitated from HeLa cells that are treated with or without $\mathrm{H}_{2} \mathrm{O}_{2}$, and then examined by Western blotting with indicated antibodies. hnRNP A1 shows both steady state and induced levels of PARylation, whereas no PARylation of TDP-43 was detected even when the blot is overexposed to facilitate the detection. c Cells expressing the Flag-tagged hnRNP A1 are treated with $\mathrm{H}_{2} \mathrm{O}_{2}$ and immunoprecipitated with the anti-Flag as in (b); PARG is added to the immunoprecipitates and then examined by Western blot with anti-Flag or anti-PAR. The bands of hnRNP A1 and the associated proteins in the anti-PAR blot are drastically decreased, confirming that they are indeed due to PARylation. d Purified, recombinant His-tagged TDP-43 $3^{1-274}$, TDP-43 $3^{274-414}$ and full-length hnRNP A1 proteins (4 $\mu \mathrm{g}$ ) were subjected to in vitro PARylation with the recombinant PARP1 and ssDNA (to activate PARP1 in vitro) as indicated. After reaction, proteins were separated on the SDS-PAGE gel. The Coomassie Brilliant Blue staining confirms equal protein loading (upper panel), followed by immunoblotting with the anti-PAR (lower panel). Activated PARP1 shows self-PARylation smears at above $115 \mathrm{kDa}$, which induces PARylation of TDP-43 ${ }^{1-274}$ and hnRNP A1 but not TDP-43 ${ }^{274-414}$. e A diagram showing the heterogeneity of PARylation, which is reversibly catalyzed by PARPs and PARG. ADPr, an ADP-ribose unit. $f$ Functional domains of the human hnRNPA1 protein. RRM RNA recognition motif, RGG RGG box RNA binding domain, M9 a nuclear targeting sequence termed M9, GRD glycine-rich domain, PBM PAR-binding motif. The consensus sequence and the residues mutated in the PBM and putative PARylation sites are indicated. $H$ hydrophobic amino acid residues, $B$ basic amino acid residues. $\mathbf{g}$ Examination of PARylation and PAR-binding of the WT, K298A and PBM ${ }^{\text {mut }}$ hnRNP A1. hnRNP A1-Flag was immunoprecipitated from HeLa cells with the antiFlag and examined by Western blotting with the anti-PAR. The blot is overexposed to facilitate the detection of less abundant PARylated proteins. * the rabbit anti-HA IgG heavy chain, \# PARylated proteins co-immunoprecipitated with hnRNP A1. h-i Representative images (h) and quantification (i) of the dot-blot assay showing significantly reduced binding affinity of the PBM ${ }^{\text {mut }}$ to the PAR polymer in vitro. The MBP was used as a negative control. The PAR intensity was normalized to that of the Ponceau S staining (loading control) and shown as the percentage to the WT hnRNP A1 in (i). Mean \pm SEM, $n=3,{ }^{* * *} p<0.001$; Student's $t$-test

override the regulation of nucleocytoplasmic shuttling by K298 PARylation, leading to abnormal cytoplasmic accumulation of hnRNP A1 in ALS patients.

PAR promotes the phase separation of hnRNP A1

Disease-related RBPs such as hnRNP A1 can phase separate in vitro, which may be involved in the regulation of the assembly of RNP granules and the pathological progression to amyloid aggregations in vivo. ${ }^{4}$ The findings that hnRNP A1 could bind to PARylated proteins and PAR (Fig. 2) and that the PAR-binding capability affected its SG association (Fig. 3) prompted us to test whether PAR could directly regulate the LLPS of hnRNP A1. As previously reported, ${ }^{4}$ recombinant hnRNP A1 formed dynamic liquid droplets (LDs) in vitro, which increased in size with decreasing salt concentrations $(\mathrm{NaCl}, 25-300 \mathrm{mM})$ and increasing hnRNP A1 concentrations (10-60 $\mu \mathrm{M})$ (Fig. 4a).

Next, to determine if PAR affected the LLPS of hnRNP A1, we chose a condition at which spontaneous LLPS of hnRNP A1 barely occurred $(20 \mu \mathrm{M}$ hnRNPA1 and $25 \mathrm{mM} \mathrm{NaCl}$, Fig. 4a). Briefly, increasing concentrations of PAR polymers $(1-7 \mu \mathrm{M})$ were added to the in vitro demixing system, which induced the LLPS of hnRNP A1 in a dose-dependent manner (Fig. 4b). As a control, addition of heparin $(7 \mu \mathrm{M})$ did not promote the LLPS of hnRNP A1 (Fig. 4c). Similarly, PAR $(7 \mu \mathrm{M})$ alone did not phase separate in vitro at the same condition (Fig. 4d). Therefore, the effect of PAR to promote LLPS of hnRNP A1 is rather specific. Further, the effect of PAR in promoting the LLPS was only observed with WT hnRNP A1 but not the PAR-binding deficient PBM $^{\text {mut }}$ (Fig. 4e), confirming that this 
a

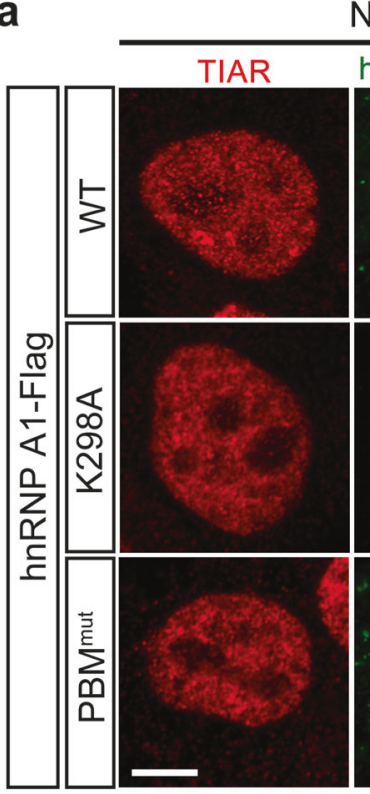

No stress (PBS)

hnRNP A1-Flag
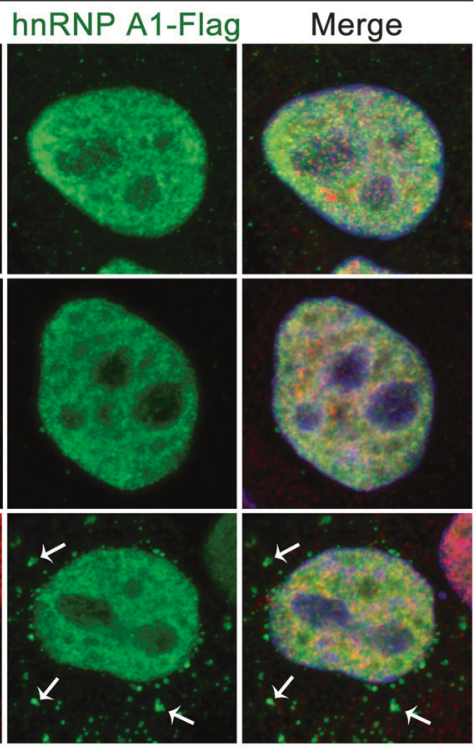

C
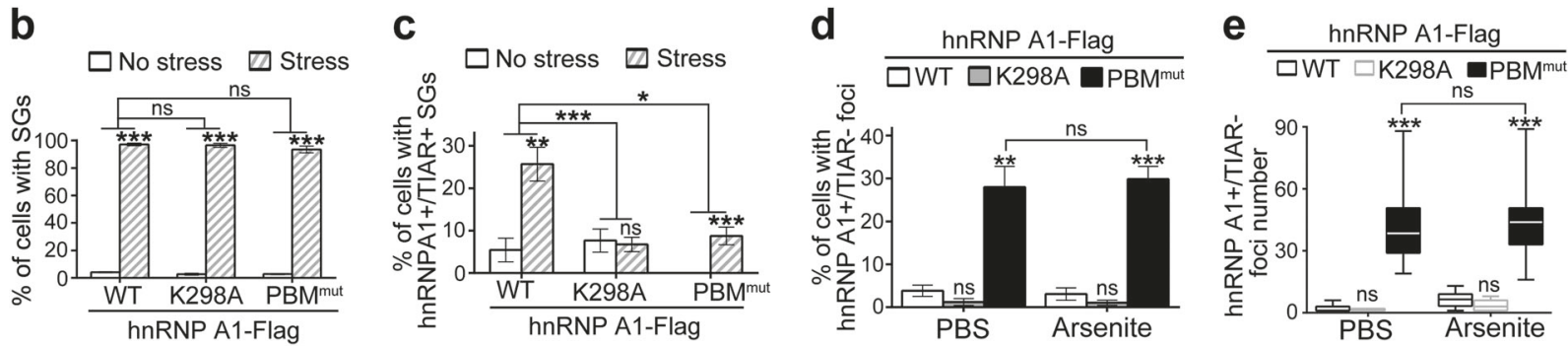

Fig. 3 The PARylation and PAR-binding mutants of hnRNP A1 K298A and PBM ${ }^{\text {mut }}$ exhibit deficits in translocation and targeting to SGs. a Representative images of HeLa cells transfected with the WT, K298A or PBM ${ }^{\text {mut }}$ of the Flag-tagged hnRNP A1 and treated with PBS (no stress) or arsenite (stress) for 30 min. Arrows, hnRNP A1 + /TIAR- cytoplasmic foci; arrowheads, hnRNPA1 + /TIAR + SGs; *, hnRNP A1-/TIAR + SGs. b, c The percentage of cells showing SGs (TIAR +) (b) or SGs co-localized with hnRNP A1 (hnRNP A1 + /TIAR +) (c) is quantified. d, e The occurrence of abnormal hnRNP A1 cytoplasmic foci is assessed as the average percentage of cells showing hnRNP A1 + /TIAR- foci (d). The average count of hnRNP A1 + /TIAR- foci per cell was evaluated in such cells (e). Mean \pm SEM except for (e), for which the box-and-whisker plots are displayed; $n=$ over 300 cells for each group, pooled results of three independent repeats; ${ }^{*} p<0.05,{ }^{* *} p<0.01,{ }^{* * *} p<0.001$; ns, not significant; Student's t-test for comparison between stress and no stress within the same genotype, and two-way ANOVA for comparison of the stress-induced changes across different genotypes. Scale bar $5 \mu \mathrm{m}$

modifying effect required the PAR-binding capability of hnRNP A1. Moreover, to test whether PAR also impacted the dynamics of the LDs after their formation, we conducted the fluorescence recovery after photobleaching (FRAP) assay to examine the kinetics of diffusion in the LDs in vitro (Fig. 4f). Indeed, with the addition of PAR $(7 \mu \mathrm{M})$, the fluorescence recovery of the hnRNP A1 droplets after photobleaching was significantly delayed (Fig. 4f, g), suggesting that binding to PAR reduced the dynamics of hnRNP A1 LDs.

hnRNP A1 and TDP-43 co-phase separate in vitro and PAR promotes the process

A recent study showed that hnRNP A2, another hnRNP family protein, could co-phase separate with TDP-43 and induced coaggregation. $^{12}$ Therefore, we examined whether hnRNP A1 and TDP-43 could co-phase separate in vitro and whether PAR could influence this process. As shown in Fig. 5a, hnRNP A1 did not phase separate on its own at the condition of $50 \mu \mathrm{M}$ of hnRNP $\mathrm{A} 1$ and $100 \mathrm{mM}$ of $\mathrm{NaCl}$. Furthermore, TDP-43 $3^{1-274}(50 \mu \mathrm{M})$ showed mild LLPS at this condition, as only a few small LDs were spotted (Fig. 5b', $\left.c^{\prime}\right)$. Mixing hnRNP A1 with TDP-43 $3^{1-274}(50 \mu \mathrm{M})$ but not BSA $(50 \mu \mathrm{M})$, resulted in the formation of larger LDs (Fig. 5b, c), suggesting the co-LLPS of hnRNP A1 and TDP$43^{1-274}$. While TDP- $43^{274-414}$ was more prone to phase separation even at a lower concentration (20 $\mu$ M; Fig. $\left.5 d^{\prime}, e^{\prime}\right)$, the addition of TDP- $43^{274-414}$ did not promote phase separation of hnRNPA 1 at the same condition used for TDP-43 ${ }^{1-274}$ (Fig. $5 d$, e). However, the co-LLPS of hnRNP A1 with TDP-43 $3^{274-414}$ could be observed when adding a commonly used crowding reagent PEG $3550(10 \%, v / w)$ to the in vitro demixing system (Supplementary information, Fig. S6). This result was consistent with a previous report suggesting hnRNP A2 co-phase separates with the C-terminus of TDP-43. ${ }^{12}$

To further verify that hnRNP A1 and TDP-43 $3^{1-274}$ indeed cophase separated, rather than the LLPS of one protein was enhanced by the other, we prepared fluorophore-labeled hnRNP A1 and TDP-43 ${ }^{1-274}$ proteins. hnRNP A1-Alexa 647 alone did not form any LDs, while the TDP-43 ${ }^{1-274}$-Alexa 555 formed a few small, green LDs. When the two proteins were mixed, they formed large LDs that contained both hnRNP A1 and TDP-43 ${ }^{1-274}$ (Fig. 5f). The dose-dependent co-LLPS of hnRNP A1 and TDP-43 $3^{1-274}$ was further established in Supplementary information, Fig. S7a. Together, our results indicated that hnRNPA1 could co-phase separate with TDP-43 $3^{1-274}$ in vitro.

We then examined whether PAR affected the co-LLPS of hnRNP A1 and TDP-43. To test this, we lowered the concentration of hnRNP A1 and TDP-43 $3^{1-274}$ to $12.5 \mu \mathrm{M}$ each and $\mathrm{NaCl}$ to $50 \mathrm{mM}$, at which spontaneous co-LLPS did not occur (Fig. $5 \mathrm{~g}$ ). The addition 


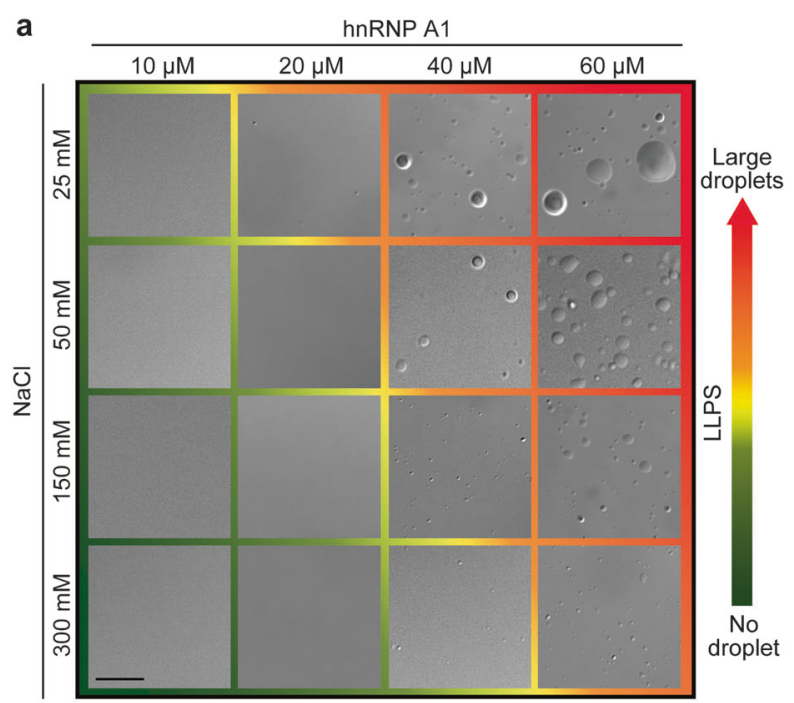

C

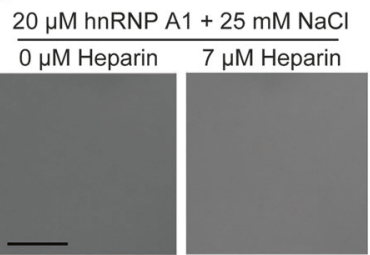

d $25 \mathrm{mM} \mathrm{NaCl}$

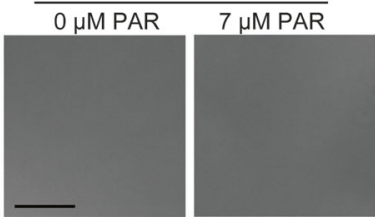

f

$20 \mu \mathrm{M}$ hnRNP A1 $+25 \mathrm{mM} \mathrm{NaCl}$
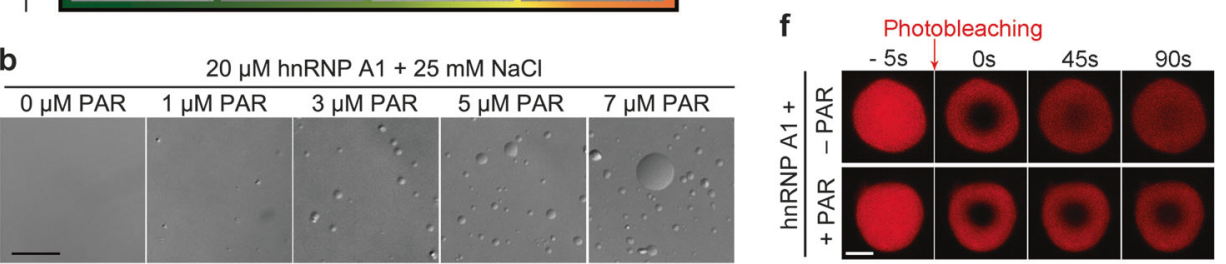
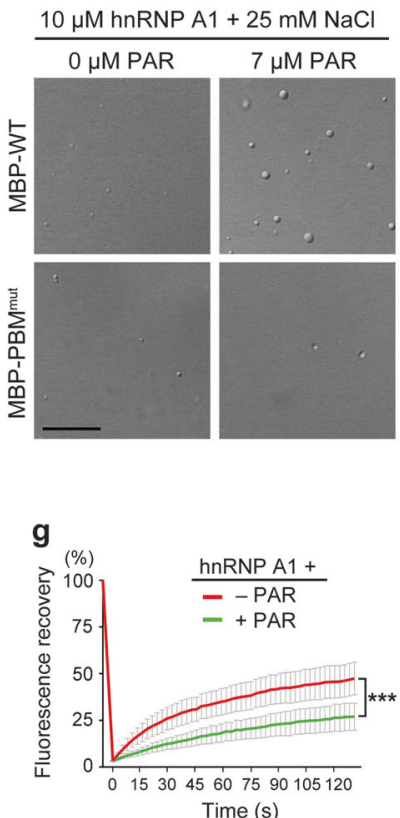

Fig. 4 PAR promotes LLPS of hnRNPA1 in vitro. a hnRNPA1 forms dynamic LDs by LLPS in vitro. The concentrations of hnRNP A1 and NaCl are shown. The higher hnRNP A1 and lower NaCl concentrations, the more and larger LDs are formed. $b$ Addition of PAR promotes LLPS of hnRNP A1 in a dose-dependent manner. c The same concentration of heparin $(7 \mu \mathrm{M})$ does not induce LLPS of hnRNP A1 at the same condition. d PAR $(7 \mu \mathrm{M})$ alone does not phase separate in vitro. e Addition of PAR in the demixing system promotes LLPS of the WT but not the PAR-binding deficient PBM ${ }^{\text {mut }}$ hnRNP A1. f Representative images of the FRAP analysis of Alexa 647-conjugated hnRNP A1 LDs in the absence or presence of $7 \mu \mathrm{M}$ of PAR in vitro. $\mathbf{g}$ The FRAP recovery curve by averaging the signals of $5 \sim 6$ droplets per group with similar sizes after photobleaching. The relative fluorescence intensity of each droplet prior to photobleaching was set to $100 \%$. Time 0 refers to the time point right after photobleaching. Mean \pm SEM, ${ }^{* * *} p<0.001$; two-way ANOVA. Scale bars $10 \mu \mathrm{m}$ in (a-e) and $2 \mu \mathrm{m}$ in (f)

of PAR $(1 \sim 7 \mu \mathrm{M})$ promoted co-LLPS of hnRNP A1 and TDP-43 ${ }^{1-274}$ in a dose-dependent manner, whereas addition of heparin $(7 \mu \mathrm{M})$ had no such effect (Fig. $5 \mathrm{~g}$ ). The fluorescent microscopy-based assay confirmed that the LDs triggered by the addition of PAR contained both hnRNP A1 and TDP-43 $3^{1-274}$ (Fig. 5h). The effect of PAR on the co-LLPS was further quantified using a semiquantitative, centrifugation-based assay ${ }^{43}$ (Supplementary information, Fig. S7b, c). PAR could not promote the co-LLPS of the PBM $^{\text {mut }}$ hnRNP A1 and TDP-43 ${ }^{1-274}$ (Fig. 5i), suggesting that this regulation is dependent on the PAR-binding capability of hnRNP A1. As a control, no LLPS was observed when PAR $(7 \mu \mathrm{M})$ was added to hnRNP A1 alone or TDP- $43^{1-274}$ alone (Fig. 5j). At the same condition, PAR $(7 \mu \mathrm{M})$ did not promote co-LLPS of hnRNP A1 with TDP-43 $274-414$ (Fig. 5k).

PAR-binding modulates the interaction between hnRNP A1 and TDP-43

To investigate how PARylation and PAR-binding influenced the interaction between hnRNP A1 and TDP-43 in vivo, we conducted the co-IP experiments. We showed that the endogenous TDP-43 protein could be co-immunoprecipitated with transiently expressed hnRNP A1-Flag and the interaction between the two proteins depended on the presence of RNA (Supplementary information, Fig. S8). These results were consistent with the previously reported interaction between TDP-43 and the hnRNP family proteins as well as their pivotal role in regulating RNA. ${ }^{29,32,44,45}$ Next, we treated the immunoprecipitates with PARG to examine whether the removal of PAR affected their binding. Indeed, we found that the PARG treatment drastically reduced the amount of TDP-43 co-immunoprecipitated with hnRNP A1 (Fig. 6a, b). On the other hand, activation of PARP1 by $\mathrm{H}_{2} \mathrm{O}_{2}$ together with
PARG KD significantly increased the PARylation levels of hnRNP A1 and the amount of TDP-43 co-immunoprecipitated with hnRNP A1 (Fig. 6c, d).

The PARG treatment and the PARP1 activation experiments indicated that the PARylation levels modulated the interaction between hnRNP A1 and TDP-43. We next investigated whether the PARylation or the PAR-binding of hnRNP A1 mediated this regulation. We examined how well WT, K298A and $\mathrm{PBM}^{\text {mut }}$ hnRNP A1 could pull down TDP-43 in the co-IP experiments. Compared to that of WT hnRNP A1, the PARylation mutant K298A showed a similar level of co-IP of TDP-43. In contrast, the PAR-binding deficient $\mathrm{PBM}^{\text {mut }}$ hnRNP A1 showed dramatically reduced association with TDP-43 (Fig. 6e, f). Of note, the protein levels of PBM ${ }^{\text {mut }}$ in the input were lower due to reduced solubility (Supplementary information, Fig. S9). To compare the co-IP efficiency with a similar input level, we transfected cells with 2 times of the $\mathrm{PBM}^{\text {mut }}$ expression plasmids $\left(2 \times \mathrm{PBM}^{\mathrm{mut}}\right)$. The input protein levels of the $\mathrm{PBM}^{\text {mut }}$ were significantly improved, however, the co-IP of TDP-43 was still barely detected (Fig. 6e, f). Overall, these data indicate that PAR-binding via the PBM of hnRNP A1 modulates the interaction between hnRNP A1 and TDP-43.

Inhibition of PARylation reduces the cytotoxicity of hnRNP A1 and TDP-43 in motor neuron-like NSC-34 cells

We extended the study of PARylation in the regulation of the biochemical properties of the disease-associated RBPs to their functional readouts such as the cytotoxicity. We used the mouse motor neuron (MN)-like hybrid cell line, NSC-34 cells, to overexpress hnRNP A1 or TDP-43 by lentiviral infection. The cells overexpressing hnRNP A1 or TDP-43 exhibited remarkable morphological changes and a significant reduction of cell viability 


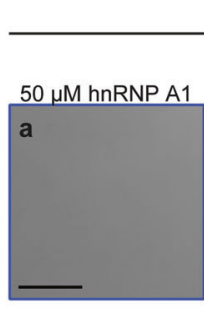

$\begin{array}{rc}100 \mathrm{mM} \mathrm{NaCl} \\ 50 \mu \mathrm{M} \text { BSA } & 50 \mu \mathrm{M} \text { TDP-43 } \\ & \text { 1-274 }\end{array}$

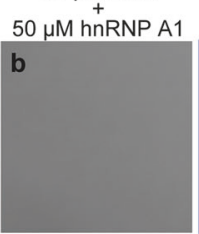

$50 \mu \mathrm{M} B S A$
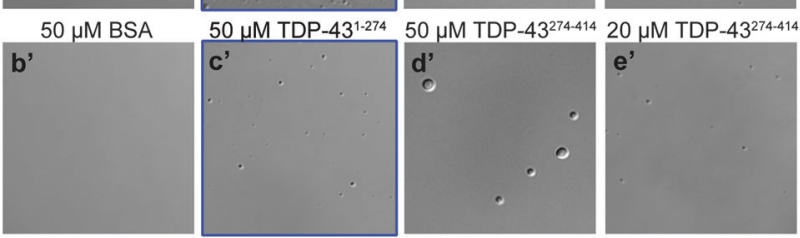

g

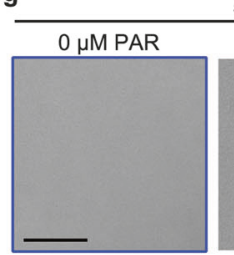

$50 \mathrm{mM} \mathrm{NaCl}+12.5 \mu \mathrm{M}$ hnRNP A1 + $12.5 \mu \mathrm{M}$ TDP-43 $3^{1-274}$
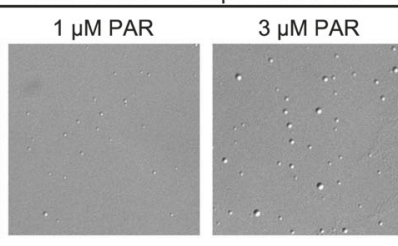

$7 \mu \mathrm{M}$ PAR
$\vdots$
$\vdots$
$\vdots$
$\vdots$

i

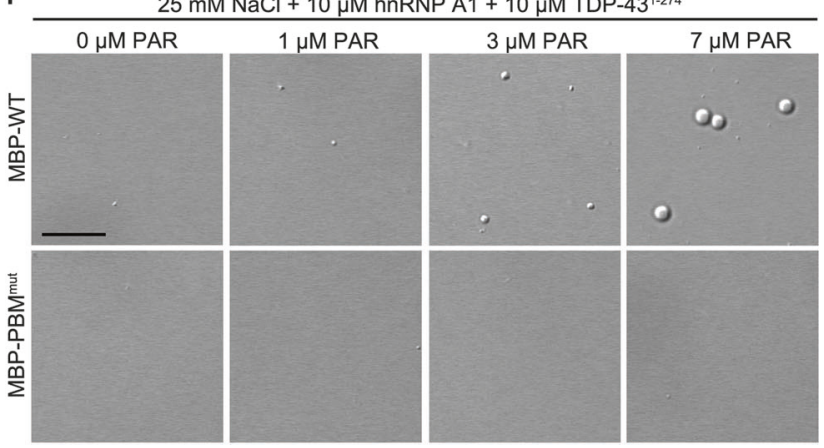

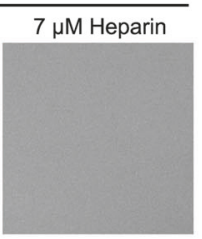
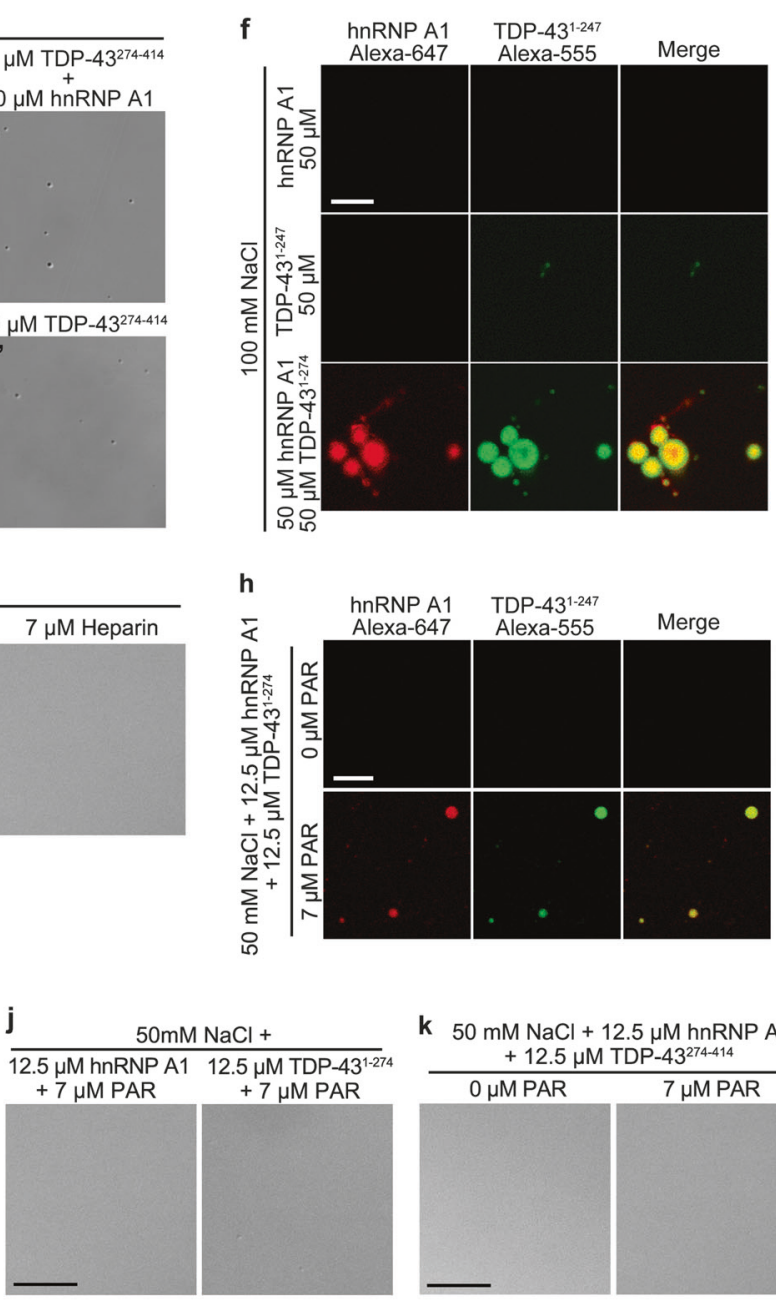

k $50 \mathrm{mM} \mathrm{NaCl}+12.5 \mu \mathrm{M}$ hnRNP A1

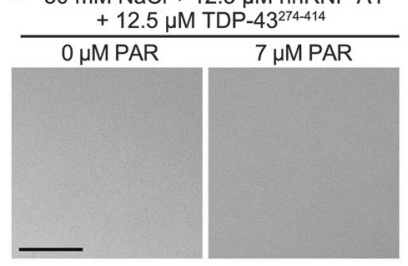

Fig. 5 hnRNP A1 and TDP-43 co-phase separate in vitro and PAR affects this process. a hnRNP A1 alone does not form spontaneous LDs by LLPS at the indicated condition in vitro. b-e co-LLPS experiments of hnRNP A1 with BSA (b), TDP-43 ${ }^{1-274}$ (c), or TDP-43 ${ }^{274-414}$ (d-e) with the indicated concentrations of each protein. $\mathbf{b}^{\prime}-\mathbf{e}^{\prime}$ LLPS experiments of BSA $\left(\mathbf{b}^{\prime}\right)$, TDP-43 ${ }^{1-274}\left(\mathbf{c}^{\prime}\right)$, or TDP-43 ${ }^{274-414}\left(\mathbf{d}^{\prime}-\mathbf{e}^{\prime}\right)$ alone at the same condition as in (b-e). f Fluorophore-conjunct hnRNP A1 (Alexa Fluor-647, red) and TDP-43 ${ }^{1-247}$ (Alexa Fluor-555, green) confirms that the LDs formed by the co-LLPS contain both the proteins. g PAR promotes the in vitro co-LLPS of hnRNP A1 and TDP-43 ${ }^{1-274}$ in a dose-dependent manner. Heparin $(7 \mu \mathrm{M})$ does not have such an effect. h PAR $(7 \mu \mathrm{M})$ promotes the co-LLPS of hnRNP A1 (Alexa Fluor-647, red) and TDP-43 ${ }^{1-247}$ (Alexa Fluor-555, green), as the LDs contain both red and green fluorescence. i PAR $(7 \mu \mathrm{M})$ does not promote co-LLPS of PBM ${ }^{\text {mut }}$ hnRNP A1 with TDP-43 ${ }^{1-274}$. $\mathbf{j}$ At the same condition of $(\mathbf{g})$, PAR does not trigger the LLPS of hnRNP A1 or TDP-43 ${ }^{1-274}$ alone. $\mathbf{k}$ Addition of PAR does not promote the in vitro co-LLPS of hnRNPA1 and TDP-43 ${ }^{274-414}$. The conditions highlighted by a blue box in (a-e') and (g) are examined in (f) and (h), respectively. The concentration of each component in the demixing system is indicated. Fluorescent images are shown in pseudo colors. Scale bars $5 \mu \mathrm{m}$ in (f) and (h), $10 \mu \mathrm{m}$ all the others

(Fig. 7a, b and Supplementary information, Fig. S10), which confirmed the cytotoxicity of overexpression (OE) of hnRNP A1 or TDP-43 in the MN-like cell model.

Next, to examine whether lowering the overall PARylation levels in cells could mitigate the cytotoxicity of hnRNP A1 and TDP-43, we treated NSC-34 cells with the siRNA of PARP1 (si-PARP1). Downregulation of PARP1 significantly suppressed the decrease of cell viability induced by the OE of hnRNP A1 or TDP-43 (Fig. 7c-e). Consistently, PARG KD remarkably enhanced the cytotoxicity mediated by the OE of hnRNP A1 or TDP-43 (Fig. 7f-h). In addition, we examined PARP inhibitor Olaparib on TDP-43 OE-mediated cytotoxicity. We tested different doses and found that $5 \mu \mathrm{M}$ of Olaparib did not affect the viability of NSC-34 cells on its own but showed a remarkable suppression of TDP-43 OE-mediated cytotoxicity (Fig. 7i). Therefore, both the genetic and pharmacological inhibition of PARP significantly suppressed the cytotoxicity of ALS-associated RBPs in MN-like NSC-34 cells.
Downregulation of Parp or upregulation of Parg suppresses TDP43-mediated neurodegeneration in a Drosophila model of ALS Finally, we validated these findings in an in vivo model of ALS using the transgenic flies expressing human TDP-43 (hTDP-43). The expression of hTDP-43 OE in the fly photoreceptor cells (GMR driver) caused an age-dependent eye degeneration, which was drastically suppressed by transgenic downregulation of the Drosophila Parp (RNAi-Parp) (Fig. 8a, c; Supplementary information, Fig. S11a). The protein levels or solubility of hTDP-43 was not significantly affected (Supplementary information, Fig. S11b, c), confirming that the suppression by RNAi-Parp was not due to an alteration of the transgenic expression system or the abundance of the hTDP-43 protein in the flies. Consistently, the OE of the Drosophila Parg (UAS-Parg) in the fly eye also effectively suppressed the TDP-43 OE-mediated degeneration (Fig. 8b, d). Furthermore, we performed the climbing and lifespan assays to evaluate the behavioral consequences, which more closely resembled 
a

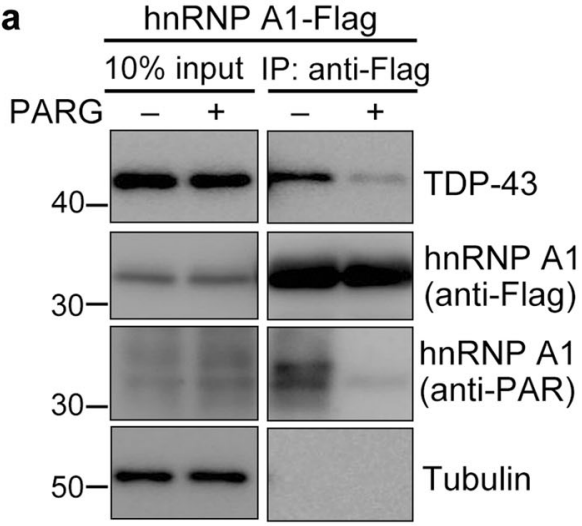

b

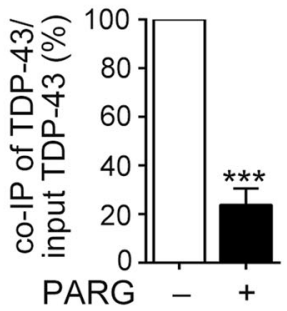

e

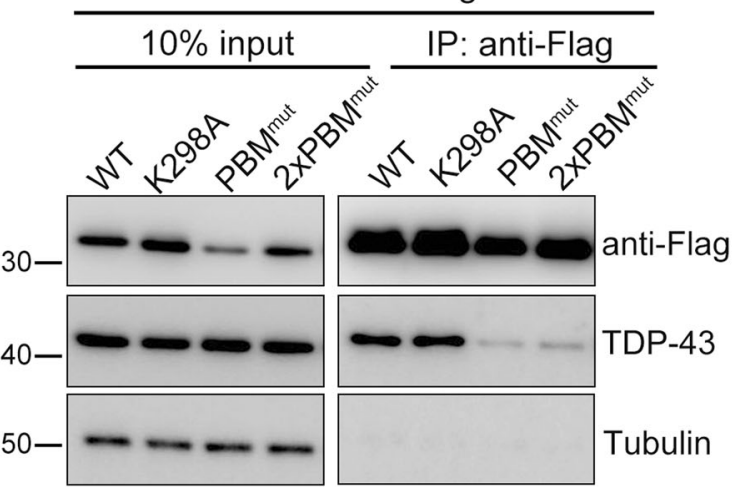

c hnRNP A1-Flag

$10 \%$ input IP: anti-Flag

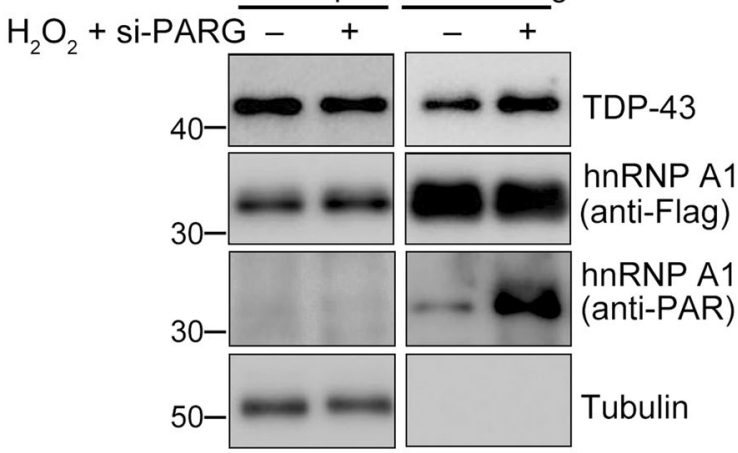

d

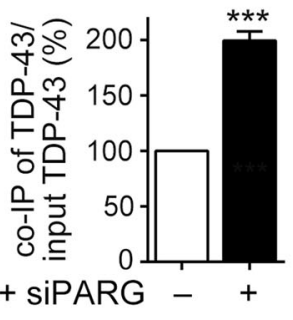

f

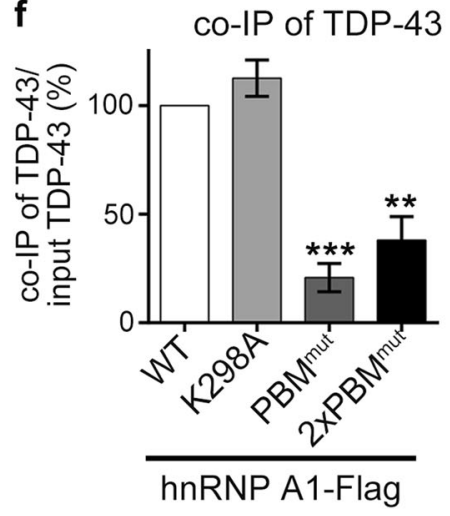

Fig. 6 PARylation modulates the interaction between hnRNP A1 and TDP-43. a, b Flag-tagged hnRNP A1 is expressed in HeLa cells and immunoprecipitated with anti-Flag. The immunoprecipitates are treated with PARG $(1 \mu \mathrm{g})$ or buffer only (control) for $1 \mathrm{~h}$ and then examined by Western blotting in (a). The level of co-IP of TDP-43 with hnRNP A1 is quantified as the relative ratio of co-IP of TDP-43/input TDP-43 and shown as the percentage to the "no PARG treatment" control group in (b). c, d Representative co-IP analysis (c) and quantification (d) of cells transfected with the hnRNP A1-Flag and treated with $\mathrm{H}_{2} \mathrm{O}_{2}$ and si-PARG to boost the PARylation levels in cells. e Cells transfected with the WT, K298A or PBM ${ }^{\text {mut }}$ of hnRNP A1-Flag were immunoprecipitated with the anti-Flag and examined by Western blotting with the indicated antibodies. $2 \times$ PBM $^{\text {mut }}$, cells transfected with 2 times of the PBM ${ }^{\text {mut }}$ expression plasmids. $f$ The relative level of TDP-43 co-immunoprecipitated with the WT or mutant hnRNP A1 protein is normalized to TDP-43 level in the input and shown as a percentage to the WT hnRNP A1 group. Means \pm SEM, $n=3 ;{ }^{* *} p<0.01,{ }^{* * *} p<0.001$; One-way ANOVA

the disease-relevant symptoms in ALS. We induced hTDP-43 OE in the adult fly neurons using an elavGS driver and added RU486 to the fly food $(80 \mu \mathrm{g} / \mathrm{mL})$ starting from day one after adult flies emerge from the pupa. Expression of hTDP-43 in the adult fly neurons caused an age-dependent decline of the climbing capability and a significant shortening of the lifespan, both of which could be suppressed by RNAi-Parp in the fly neurons (Fig. 8e, f). Together, these data indicate that manipulation of PARP or PARG levels can modify TDP-43-mediated neurodegeneration in vivo.

\section{DISCUSSION}

PARylation regulates the SG dynamics and the phase separation of ALS-linked RBPs

In this study, we reveal that decreases in PARylation levels suppress the formation of SGs and the recruitment of hnRNP A1 and TDP-43 to SGs, while increases in the PARylation levels delay the disassembly of SGs and the recovery of the RBPs. Interestingly, the function of PARylation appears to be specifically enriched in the regulation of RNA/DNA-binding proteins and the associated complexes. For example, PARylation is involved in the regulation of the mitotic spindle, ${ }^{46}$ Cajal bodies, ${ }^{47}$ DNA damage repair, ${ }^{48}$ and microRNA-mediated translational repression. ${ }^{26}$ Given the essential role of RNP granules in RNA processing and homeostasis, ${ }^{30,44}$ dysregulation of RNP granules contributes to the pathogenesis of neurodegenerative diseases. ${ }^{49-51}$ This is at least in part because many of the disease-linked RBPs can undergo spontaneous selfassembly via LLPS to generate higher-order structures such as solidified LDs and irreversible amyloid fibrils.

The hnRNP family proteins constitute important components of the SGs and are highly regulated at both the molecular and cellular levels. For example, pathogenic mutations in the GRD of 
a

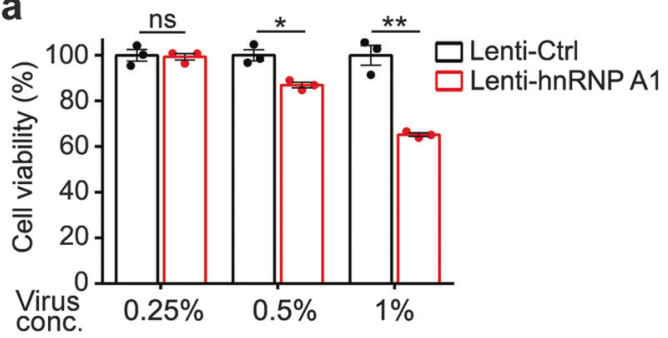

b

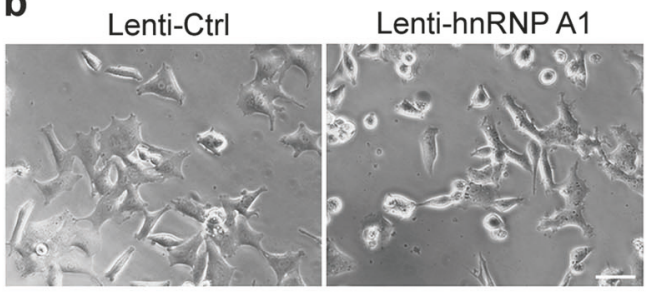

C

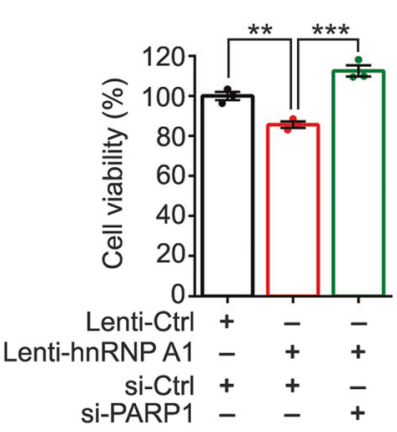

g

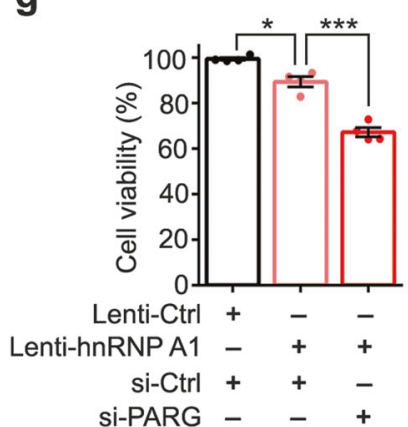

d

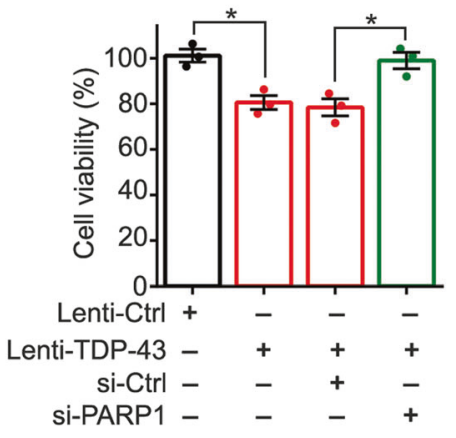

h

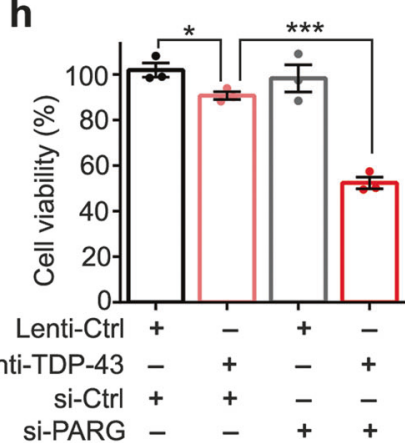

e

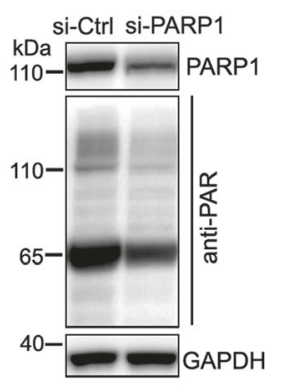

$f$

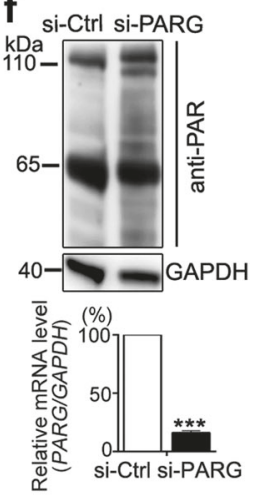

i

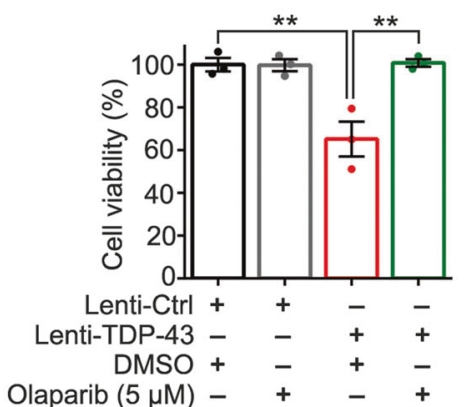

Fig. 7 PARP inhibition mitigates the cytotoxicity of hnRNP A1 and TDP-43 in MN-like NSC-34 cells. a Mouse NSC-34 cells infected with increased concentrations of lenti-hnRNP A1-HA show decreased cell viability in the Cell Counting Kit-8 (CCK-8) assay, indicating that hnRNP A1-induced cytotoxicity is dose-dependent. b Representative bright field images of NSC-34 cells infected with the Lenti-Ctrl or the LentihnRNP A1-HA (1\% virus concentration) are shown. Scale bar: $50 \mu \mathrm{m}$. c, d NSC-34 cells overexpressing hnRNP A1 (c) or TDP-43 (d) by lentivirus infection show decreased cell viability in the CCK-8 assay, which is significantly suppressed by PARP1 KD (si-PARP1) compared to the scrambled siRNA control (si-Ctrl). e Western blot analysis confirming the downregulation of PARP1 and the decrease in the overall PARylation levels by si-PARP1. f Western blot analysis (upper panel) confirms the increase of the overall PARylation levels by si-PARG; the anti-PARG antibody does not recognize the mouse PARG, the qPCR analysis (lower panel) was conducted to examine the KD efficiency of si-PARG. $\mathbf{g}$, $\mathbf{h}$ PARG KD (si-PARG) significantly enhances the cytotoxicity mediated by OE of hnRNP A1 (g) or TDP-43 (h). i The PARP inhibitor Olaparib rescues TDP-43 OE-induced reduction of cell viability in the NSC-34 model. Mean \pm SEM and the value of each repeat are shown; $n=3 ;{ }^{*} p<0.05,{ }^{* *} p<$ $0.01 ;{ }^{* * *} p<0.001 ; \mathrm{ns}$, not significant; Student's $t$-test

hnRNP A1 accelerate its recruitment to SGs and the fibrillation, ${ }^{27}$ while methylation of hnRNP A2 in the RGG domain suppresses the phase separation of hnRNP A2. ${ }^{12}$ In this study, we show that PAR promotes the LLPS of hnRNP $A 1$ in vitro and this regulation depends on the PAR-binding capability of hnRNP A1, as PAR does not promote the LLPS of the PBM ${ }^{\text {mut }}$. hnRNP A1 can form protein complexes with TDP-43 and other RNPs to regulate RNA processing. ${ }^{29,32}$ Indeed, we find that hnRNP A1 and TDP-43 can co-phase separate in vitro and PAR-binding strongly regulates their interaction in vitro and in cells. Consistently, during the manuscript preparation of this study, McGurk and colleagues independently reported that the liquid demixing of TDP-43 could be affected by PAR. ${ }^{52}$ Considering that the proposed PBM is situated within or near the RRMs in hnRNP A1 and TDP-43, the PAR-binding at these topological regions may also participate in the modulation of the RNA-binding affinity as well as the RNAmediated protein-protein interactions.

In this study we demonstrate that PARP1 can PARylate hnRNP $A 1$ and regulate the basal PAR levels in the cell so that the administration of the PARP inhibitor or the PARP1 siRNA affects the SG association and cytotoxicity of hnRNP A1. It is important to note that PARP1 accounts for $\sim 90 \%$ of the ADP-ribosyl transferase activity in cells ${ }^{53}$ and shares the predominant nuclear localization with hnRNP A1. Nevertheless, our data do not exclude the possibility that the translocated hnRNP A1 protein may be further modified by cytoplasmic PARP(s), such as by tankyrase/PARP5 as proposed for TDP-43. ${ }^{52}$ Together, PARylation may serve as an important regulator of the dynamics of the RNP granules, where in case of lasting stimuli or excessive PARylation, pathological irreversible RNP aggregates may form (Fig. 8g). 
a
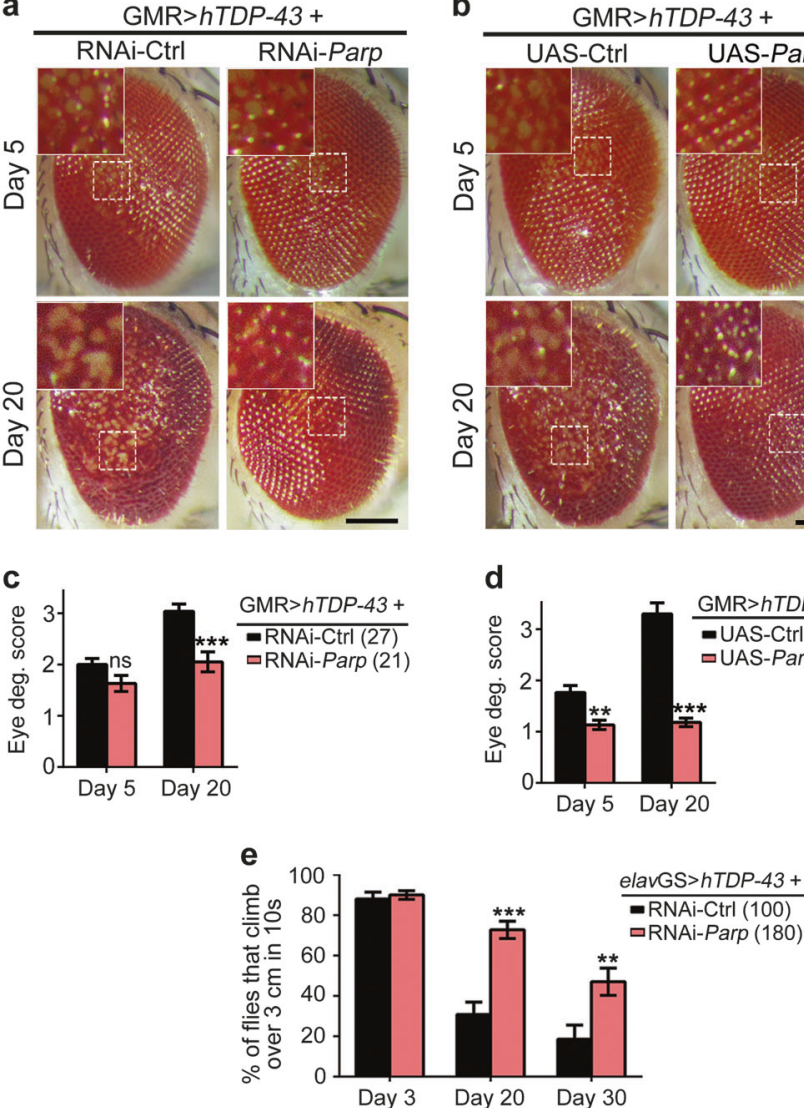

b

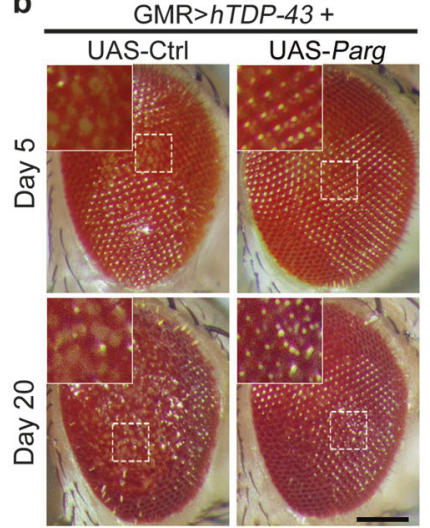

d

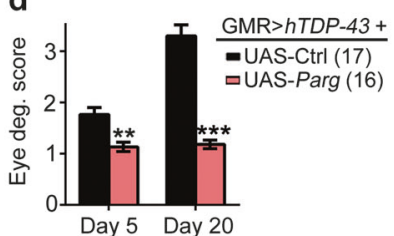

f
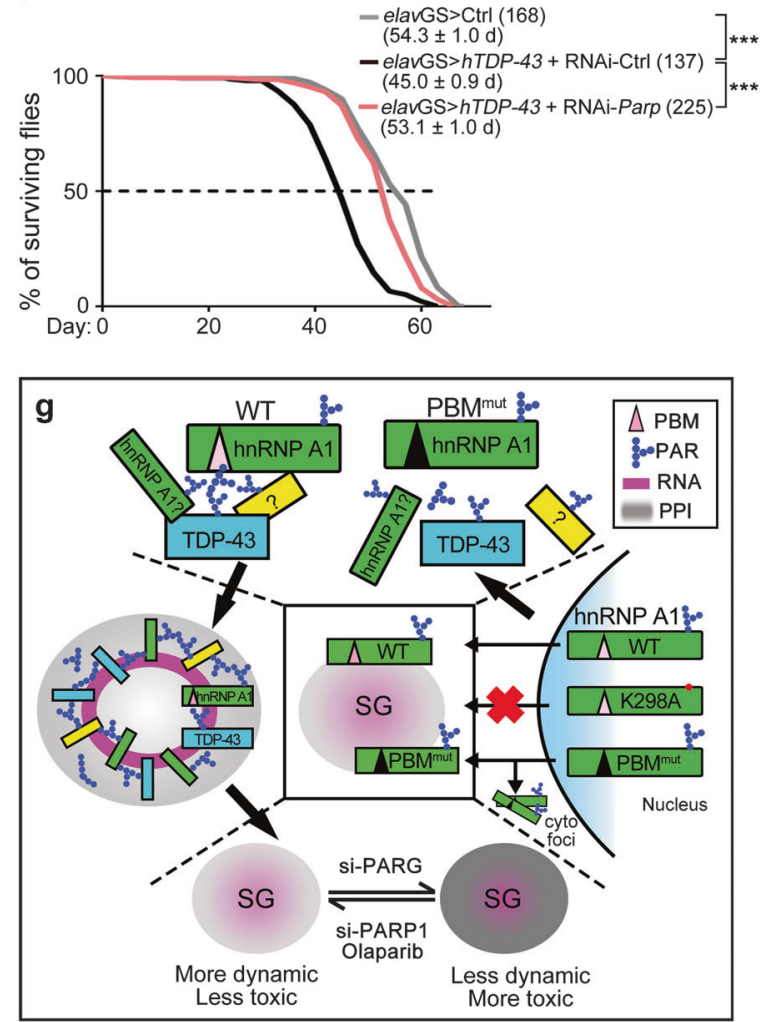

Fig. 8 Downregulation of Parp or upregulation of Parg alleviates neurodegeneration in a Drosophila model of ALS. a-d Representative images $(\mathbf{a}, \mathbf{b})$ and quantifications of the degeneration scores (c, d) of the fly eyes (GMR-Gal4) expressing hTDP-43 with RNAi-Parp or RNAi-Ctrl (RNAi$m$ Cherry) (a, c) or UAS-Parg or UAS-Ctrl (UAS-lacZ) (b, d). e, f RNAi KD of Parp in adult fly neurons using an elavGS driver (induced with RU486, $80 \mu \mathrm{g} / \mathrm{mL}$, starting from day one of adulthood) alleviates the TDP-43-mediated climbing decline (e) and the lifespan shortening (f). The climbing capability is evaluated as the average percentage of flies that climb over $3 \mathrm{~cm}$ within $10 \mathrm{~s}$. The median lifespan and the number of flies tested for each genotype are indicated. UAS-Ctrl, UAS-LacZ; RNAi-Ctrl, UAS-RNAi-mCherry. Mean \pm SEM; ${ }^{* *} p<0.01$, ${ }^{* * *} p<0.001$; ns, not significant; Student's t-test. Scale bar $100 \mu \mathrm{m}$. g A schematic model of the role of PARylation in regulating SGs and disease-related RNPs. hnRNPA1 is PARylated at K298 and binds to PAR and/or PARylated proteins via the PBM. PARylation and PAR-binding differentially regulate the nuclear export and SG association of hnRNPA1 - the K298A mutant does not respond to stress and remains in the nucleus; the PBM ${ }^{\text {mut }}$ can be recruited to SGs, but a portion of it forms mis-localized cytoplasmic foci that do not co-localize with SGs. While the formation of the RNP granules requires RNAs, the overall PARylation levels modulate the protein-protein interactions between the RBPs as well as the assemblydisassembly dynamics of the SGs. PARylation, either in the form of free PAR polymers or as PAR chains from the PARylated proteins, may act as a molecular glue to promote LLPS and enhance the assembly of SGs by cross-linking different RBPs in the granules. Pharmacological or genetic inhibition of PARP1 improves the dynamics of RNP granules and mitigates the hnRNP A1 and TDP-43 OE-mediated neurotoxicity. PAR, poly(ADP-ribose); PBM, PAR-binding motif; PPI, protein-protein interaction; WT, wild-type hnRNP A1; K298A, PARylation site mutant of hnRNP $\mathrm{A} 1 ; \mathrm{PBM}^{\text {mut }}$, PAR-binding motif mutant of hnRNP A1; SG, stress granule; cyto foci, abnormal cytoplasmic foci

Dual regulation of hnRNP A1 by PARylation and PAR-binding In this study, we show that hnRNP A1 contains a PARylation site at K298 in the GRD and a PBM that resides between the two RRMs. We further reveal that the K298 PARylation regulates the nucleocytoplasmic transport of hnRNP A1, whereas PAR-binding modulates the LLPS of hnRNP A1 in vitro and the SG association in vivo. The $\mathrm{K} 298$ is localized at the C-terminus of the M9 domain, which is a non-classical NLS of hnRNP A1. Of note, the K298 is not within the M9 core sequence (SNFGPMKGGNFGGRSSGPY), which is crucial for the nuclear import and the binding to Transportin. ${ }^{54}$ Indeed, the PARylation mutant K298A did not show any defect in either the nuclear localization or the SG association following its translocation to the cytoplasm, e.g., by a disease-linked P288A mutation (see Supplementary information, Fig. S5). In contrast, its cytoplasmic translocation in response to stress is impeded, suggesting that PARylation at the K298 may be an important mechanism for hnRNP A1 to sense stress and/or serve as a nuclear export signal. Excessive nuclear accumulation of ALS-linked RBPs can be pathogenic; for example, the nuclear TDP-43 was previously reported to cause toxicity. ${ }^{55}$

The PAR-binding deficient PBM ${ }^{\text {mut }}$ of hnRNP A1 can respond to stress and translocate to $S G s$, likely because the regulation of the nuclear export by PARylation at the K298 is intact or perhaps even increased (see below). However, the SG recruitment of the PBM ${ }^{\text {mut }}$ is less effective than that of the WT and a significant portion of $\mathrm{PBM}^{\text {mut }}$ forms mis-localized cytoplasmic foci that do not colocalize to SGs. Of note, the $\mathrm{PBM}^{\text {mut }}$ protein showed hyperPARylation possibly at the K298 of hnRNP A1 (see Fig. 2g), which may result in an abnormal activation of the nuclear export of the $\mathrm{PBM}^{\text {mut }}$ protein. On the other hand, since the $\mathrm{PBM}^{\text {mut }}$ shows a greatly reduced capacity in binding to other PARylated proteins and PAR, we speculate that the association with PARylated proteins and/or the PAR-mediated protein-protein interactions may ensure the proper targeting and anchoring of hnRNP A1 to SGs. Moreover, the data that the increased cellular PARylation levels by PARG KD delay TDP-43 and hnRNP A1 SG disassembly and that addition of PAR decreases the dynamics of hnRNP A1 LDs 
in the in vitro assays suggest that the cellular PARylation levels may be a critical factor in the development of SGs to pathogenic aggregates. Consistent with this idea, decreases in the PARylation levels by the PARP1 KD or the PARP1 inhibitor suppress whereas increases in the PARylation levels by the PARG KD enhances the cytotoxicity of hnRNP A1 and TDP-43. Therefore, under disease conditions, a reduction of PARylation or PAR-mediated association of RNPs and the restoration of the dynamics of the RNP granules may be beneficial.

Potential therapeutic values of PARP inhibitors for neurodegenerative diseases

Small-molecule inhibitors of PARP enzymes have received increasing interests due to their effect in killing BRCA1/2-mutant cancer cells. While the involvement of PARP in DNA damage repair has heralded the development of PARP inhibitors in cancer biology and therapy, ${ }^{56}$ the PARP functions go beyond DNA repair and oncology. ${ }^{57}$ For example, the neuroprotective effects of PARP inhibitors were reported in Huntington's disease, cerebral ischemia, axonal injury, and, most recently, in Parkinson's disease. ${ }^{25,58-61}$ In this study, we show that Olaparib, an FDA approved PARP inhibitor for treating ovarian cancer and breast cancer, can significantly reduce the cytotoxicity of hnRNP A1 and TDP-43 in the motor neuron-like NSC-34 cells, likely due to the pivotal role of PARylation in regulating the protein-protein interaction and the dynamics of the disease-associated, aggregation-prone RNP granules and protein complexes (Fig. 8g). Together, we propose Olaparib as a candidate for developing therapeutics for neurodegenerative diseases such as ALS. Further animal and clinical studies are needed to evaluate the potential therapeutic value of Olaparib and other PARP inhibitors in the treatment of ALS and other related diseases.

\section{MATERIALS AND METHODS}

Plasmids and constructs

To generate pCAG-TDP-43-HA, pCMV-TDP-43-3 $\times$ Flag and pBIDUASC-TDP-43 plasmids, the human TDP-43 DNA was amplified from a TDP-43-Myc plasmid ${ }^{62}$ by PCR using the primers specified below. The desired PCR products were subcloned into a $\mathrm{PCAG}^{63}$ or a pCMV-3Tag-3B (Agilent Technologies) vector using the ClonExpress $^{\mathrm{TM}}$ II One Step Cloning Kit (Vazyme). To generate the pCAGhnRNP A1-Flag plasmid, the human hnRNP A1 coding sequence was amplified from the CDNA of HeLa cells by PCR and sub-cloned into a pCAG vector by homologous recombination using the ClonExpress $^{\mathrm{TM}}$ II One Step Cloning Kit (Vazyme). The pCAG-K298AFlag, pCAG-P288A-Flag, pCAG-P288A/K298A-Flag and pCAG$\mathrm{PBM}^{\text {mut }}$-Flag of hnRNP $A 1$ were generated by $P C R$ using the pCAG-hnRNP A1-Flag as a template and hnRNP A1 by site-directed mutagenesis using the Fast Mutagenesis Kit II (Vazyme).

For Escherichia coli (E. coli) expression, the pET-28a-TDP$43^{1-274-} 6 \times$ His and pET-28a-TDP-43 $3^{274-414-} 6 \times$ His constructs were generated by PCR amplification of the truncated TDP-43 fragments from the above full-length TDP-43 plasmid and inserted between the $\mathrm{BamHI}$ and $\mathrm{Xhol}$ sites in a pET-28a vector. The pET9d-hnRNP A1 plasmid was obtained from Addgene (Plasmid \#23026). To generate pET32M.3C-MBP-6 $\times$ His-hnRNP $A 1$, the WT or the PBM ${ }^{\text {mut }}$ hnRNP A1 sequence from the above constructs was subcloned into a pET32M.3C vector (a gift from Dr. L. Pan). To generate the lentiviral packaging constructs expressing either Flag, TDP-43-Flag or hnRNP A1-HA in NSC-34 cells, the coding sequence was amplified by PCR using the above expression plasmids as the template and each of the PCR products was sub-cloned into a pCDH-CMV-MCS-EF1-Puro vector using the restriction enzyme sites including EcoRI, Xbal, Xhol, BstBI and BamHI.
The primers used for PCR to generate the expression plasmids are summarized below. All constructs were verified by sequencing to ensure the integrity of the cloned open reading frames.

pCMV-Myc-TDP-43:

5'-ATGGCCATGGAGGCCGAATTCATGTCTGAATATATT-3'

5'-CATGTCTGGATCCCCGCGGCCGCCTACATTCCCCAGCCAGA-3'; pCMV-Myc-TDP-43 ${ }^{1-274}$ :

5'-ATGGCCATGGAGGCCGAATTCATGTCTGAATATATT-3'

5'-CATGTCTGGATCCCCGCGGCCGCCTAACTTCTITCTAACTGTCTA $\Pi \pi-3^{\prime}$

pCMV-TDP-43-Flag:

5'-CGCTCTAGCCCGGGCGGATCCCACCATGTCTGAATATATTCGGG TAAC--3'

5'-GGTATCGATAAGCTTGATATCTTCATTCCCCAGCCAGAAGA-3' pCAG-TDP-43-HA:

5'-CATCATITTGGCAAAGAATTCCACCATGTCTGAATATATTCGGGT

AAC- $3^{\prime}$

5'-GCTCCCCGGGGGTACCTCGAGTTAAGCGTAGTCTGGGACGTC

GTATGGGTACATTCCCCAG CCAGAAGACTT-3'

PCAG-TDP-43 ${ }^{1-274}-\mathrm{HA}$ :

5'-ATGGCCATGGAGGCCGAATTCATGTCTGAATATATT-3'

5'-CATGTCTGGATCCCCGCGGCCGCCTAACTTCTTTCTAACTGTCTA $\Pi T-3^{\prime}$

pCAG-hnRNP A1-Flag:

5'-CATCATITTGGCAAAGAATTCCACCATGTCTAAGTCAGAGTCTCC TAAAGAG-3'

5'-GCTCCCCGGGGGTACCTCGAGCTACTTGTCATCGTCGTCCTTGT AG-3'

pCAG-hnRNP A1 R92A-Flag:

5'-AAGAGAGCTGTCTCCGCAGAAGATTCTCAAAGACCAGGTGCCC ACTTA-3'

5'-CTITGAGAATCTTCTGCGGAGACAGCTCTCTITGGTTCCACAACT CTT-3'

pCAG-hnRNP A1 P288A-Flag:

5'-TCTGGCGCCTATGGCGGTGGAGGCCAATA-3'

5'-CGCCATAGGCGCCAGAGCTTCTGCCTCCAA-3'

pCAG-hnRNP A1 K298A-Flag:

5'-CTITGCAGCACCACGAAACCAAGGTGGCTATGGCGG-3'

5'-TTCGTGGTGCTGCAAAGTATTGGCCTCCACCGCCATAGG-3'

pCAG-hnRNPA1 KK105/106VV-Flag:

5'-AGGTGCCCACTTAACTGTGGTTGTTATATTGTTGGTGGCATTAA

AGAAGACACTGAAGAAC- $3^{\prime}$

5'-CCACCAACAAATATAACAACCACAGTTAAGTGGGCACCTGGTC TाTGAGAA-3'

pCDH-CMV-MCS-EF1-Puro-hTDP-43-Flag:

5'-GCTCTAGAGCCACCATGTCTGAATATATTCGGGTAACC-3'

5'-CCGCTCGAGCATTCCCCAGCCAGAAGACTTAG-3'

pCDH-CMV-MCS-EF1-Puro-hnRNPA1-HA:

5'-TAGAAGATTCTAGAGCTAGCGAATTCCACCATGTCTAAGTCAGA

GTCTCCTAAAGAG-3'

5'-GCAGATCCTTCGCGGCCGCGGATCCTTAAGCGTAGTCTGGGAC

GTCGTATGGGTAAAATCTTCTGCCACTGCCATAGC-3'

pCDH-CMV-MCS-EF1-Puro-Flag oligos:

5'-AATTCCTCGAGTTCGAAGCCACCATGGATTACAAGGATGACGA CGATAAGGACTATAAGGACGATGATGACAAGGACTACAAAGATGA

TGACGATAAATAGG-3'

5'-GATCCCTATTATCGTCATCATCTITGTAGTCCTTGTCATCATCG

TCCTTATAGTCCTTATCGTCGTCATCCTTGTAATCCATGGTGGCTTCGA

ACTCGAGg-3'

pET-28a-6xhis-TDP-43 ${ }^{274-414}$ :

5'-CAGCAAATGGGTCGCGCCACCGGATCCGGAAGATITGGTGGT-3'

5'-GTGGTGGTGGTGGTGCTCGAGCATTCCCCAGCCAGA-3'

pET32M.3C-MBP-6xHis-hnRNP A1:

5'-CAGGGGCCCGGATCCGAATTCATGTCTAAGTCAGAGTCTCCTAA

AGAGC-3'

5'-GTGGTGGTGGTGGTGCTCGAGTTAAAATCTTCTGCCACTGCCA-3' 
Cell culture and transfection

Both HeLa and HEK-293T cells were cultured in the Dulbecco's modified Eagle's medium (sigma, D0819) supplemented with $10 \%$ fetal bovine serum (FBS, BioWest). NSC-34 cells were cultured in the RPMI 1640 medium (Gibco, 11875-093) containing 10\% (v/v) FBS. All cells were incubated at $37^{\circ} \mathrm{C}$ in a humidified atmosphere of $95 \%$ air and $5 \% \mathrm{CO}_{2}$. PolyJet ${ }^{\mathrm{TM}}$ reagent (SignaGen, SL100688) was used for the transfection of plasmids into HeLa and HEK-293T cells. Cells were transfected for 24-72 h before proceeding with subsequent experiments. For PARP inhibitor treatments, indicated concentration of Olaparib (Selleck) was added into the medium $3 \mathrm{~h}$ before harvest or other treatments. For knockdown experiments, the siRNA (Genepharma, Shanghai) was transfected into the cells using the Lipofectamine ${ }^{\mathrm{TM}}$ RNAiMAX Transfection Reagent (Invitrogen, 13778150), according to the manufacturer's instruction. siRNA was incubated for $\sim 72 \mathrm{~h}$ for HeLa cells and $48-60 \mathrm{~h}$ for NSC-34 cells. The siRNA oligos used in this study are listed below: si-Ctrl: 5'-GCGGUGAAGUUAGAUUACATT-3'

si-hPARG: 5'-GCGGUGAAGUUAGAUUACATT-3'

si-mPARG: 5'-GCAGUUUCUUACACCUAUATT-3'

si-mPARP1: 5'-CGACGCUUAUUACUGUACUTT-3'

Stress granule induction

HeLa cells were grown on coverslips in a 12-well plate and were treated with either $100 \mu \mathrm{M}$ of $\mathrm{NaAsO}_{2}$ or PBS for $30 \mathrm{~min}$, prior to fixation with $4 \%$ paraformaldehyde. For recovery, the medium containing $\mathrm{NaAsO}_{2}$ was removed and the cells were incubated in the fresh medium for the indicated time prior to fixation. The formation of stress granules was evaluated by subsequent immunocytochemistry assays.

\section{Immunocytochemistry and confocal imaging}

After transfection and drug treatments, the cells grown on the cover slips in the 24-well plate were fixed with $4 \%$ paraformaldehyde in PBS for $15 \mathrm{~min}$ at room temperature, permeabilized in $0.5 \%$ Triton X-100 in PBS for $10 \mathrm{~min}$ and blocked with $3 \%$ goat serum in PBST (PBS $+0.1 \%$ Triton $X-100)$ for $30 \mathrm{~min}$ at room temperature. The primary and secondary antibodies in the blocking buffer were then incubated at $4{ }^{\circ} \mathrm{C}$ overnight or at room temperature for $1 \mathrm{~h}$. After 3 washes with PBST, cells were mounted on glass slides using the Vectashield Antifade Mounting Medium with DAPI (Vector Laboratories). Fluorescent images were taken with the Leica TCS SP8 confocal microscopy system using a $100 \times$ oil objective $(N A=1.4)$. Images were processed and assembled into figures using LAS X (Leica) and Adobe Photoshop CS6.

\section{Protein extraction and Western blotting}

Total protein was extracted from cells in a $2 \%$ SDS extraction buffer (50 mM Tris pH 6.8, 2\% SDS, 1\% mercaptoethanol, $12.5 \%$ glycerol and $0.04 \%$ bromophenol blue) containing the protease inhibitor cocktail (Roche, 04693132001), $20 \mu \mathrm{M}$ Olaparib (Selleck, S1060) and $8 \mu \mathrm{M}$ ADP-HPD (Millopore, 118415). To separate soluble and insoluble proteins, cultured cells or fly heads were lysed on ice in a RIPA buffer (50 mM Tris pH 8.0, $150 \mathrm{mM} \mathrm{NaCl}, 1 \%$ NP-40, $5 \mathrm{mM}$ EDTA, $0.5 \%$ sodium deoxycholate, $0.1 \%$ SDS) supplemented with protease and phosphatase inhibitors. After sonication, the homogenates were centrifuged at $13,000 \times g$ for $10-20 \mathrm{~min}$ at $4^{\circ} \mathrm{C}$. The supernatant was used as the soluble fraction and the pellets containing the insoluble fraction were dissolved in a urea buffer ( $9 \mathrm{M}$ urea, $50 \mathrm{mM}$ Tris buffer, $\mathrm{pH} 8.0$ ) after wash.

Proteins were separated using a $10 \%$ Bis-Tris SDS-PAGE (Invitrogen), immunoblotted with the primary and secondary antibodies. Detection was performed using the High-sig ECL Western Blotting Substrate (Tanon). Images were captured using an Amersham Imager 600 (GE Healthcare) and densitometry was measured using ImageQuant TL Software (GE Healthcare). The contrast and brightness were optimized equally using Adobe
Photoshop CS6 (Adobe Systems Inc.). All experiments were normalized to GAPDH or tubulin as indicated in the figures.

\section{Co-immunoprecipitation}

HeLa cells were lysed in an IP buffer $(50 \mathrm{mM}$ Tris- $\mathrm{HCl} \mathrm{pH} 7.4$, $150 \mathrm{mM} \mathrm{NaCl}, 1 \% \mathrm{NP}-40,1 \mathrm{mM}$ EDTA, 5\% glycerol) containing protease inhibitor cocktails, $20 \mu \mathrm{M}$ Olaparib and $8 \mu \mathrm{M}$ ADP-HPD. To test whether the interaction between hnRNP A1 and TDP-43 was RNA-dependent, the cell lysates were treated with $100 \mu \mathrm{g} / \mathrm{mL}$ of RNase A for 30 min (Qiagen), and then incubated with the mouse anti-Flag or the rabbit anti-HA antibody on a rotary shaker at $4{ }^{\circ} \mathrm{C}$ overnight. The mouse or rabbit lgG (Santa Cruz, sc-2025 or sc2027) was used as a control for the pull-down specificity. Anti-FLAG ${ }^{\circledR}$ M2 Affinity Gel (Sigma, A2220) or Dynabeads ${ }^{\circledR}$ Protein G beads (Novex) were then added and incubated at room temperature for $2 \mathrm{~h}$. For the PARG treatment, the anti-FLAG ${ }^{\circledR}$ M2 Affinity Gel was mixed with the cell lysates, washed twice in the IP buffer, and then incubate with $1 \mathrm{ug}$ of PARG (Sigma, SRP8023) at $37^{\circ} \mathrm{C}$ for $1 \mathrm{~h}$. The IP gels or beads were then collected, according to the manufacturer's instruction, and eluted in the $2 \times$ LDS sample buffer (Invitrogen) for the subsequent Western blotting assay.

Antibodies

The following antibodies were used for Western blotting, immunoprecipitation and immunocytochemistry assays: mouse anti-FLAG (Sigma, F3165), mouse anti-HA (Proteintech, 66006-1), anti-pan-ADP-ribose binding reagent (Millipore, MABE1016), rabbit anti-HA (CST, 3724T), rabbit anti-TDP-43 (Proteintech, 10782-2$A P)$, rabbit anti-TIAR (CST, 8509S), mouse anti-G3BP (BD, 611127), rabbit anti-hnRNPA1 (CST, 8443S), rabbit anti-PAR (ENZO, ALX210-890A-0100), mouse anti-GAPDH (Proteintech, 60004-1) and mouse anti-Tubulin (MBL, PM054). HRP conjugated secondary antibodies: goat anti-mouse (Sigma, A4416) and goat anti-rabbit (Sigma, A9169). Fluorescent secondary antibodies: goat antimouse-Alexa Fluor 488 (Life Technologies, A11012); goat antirabbit-Alexa Fluor 568 (Life Technologies, A11012).

RNA extraction and real-time quantitative PCR (qPCR)

Total RNAs were extracted from either the cultured cells or the whole fly heads (40 heads per group) using the TRIzol (Invitrogen), according to the manufacturer's instructions. Following the DNase (Promega) treatment to remove the genomic DNA, reverse transcription was performed with the High-Capacity cDNA Reverse Transcription Kit (Applied biosystems). The CDNA was then used in the real-time PCR experiment using the SYBR Green qPCR Master Mix (Bimake) with the QuantStudio ${ }^{\text {TM }} 6$ Flex Real-Time PCR system (Life Technologies). The actin mRNA levels were used as an internal control to normalize the mRNA levels of the genes of interest. The primers for qPCR are listed below:

mPARG: 5'- AGCCTCTGACACGCTTACAC-3'; 5'- CAGTCACA CCACCTCCAACA-3'

mGAPDH: 5'- CACCATCTTCCAGGAGCGAG-3'; 5'- CCTTCTCCATG GTGGTGAAGAC-3'

dParp: $5^{\prime}$-ATGAAGTACGGAGGCCAACC-3'; 5'-TCTTCACCTGACGC AAACCA-3'

dActin: 5'-GAGCGCGGTTACTCTITCAC-3'; 5'-GCCATCTCCTGCTCA AAGTC-3'

Purification of TDP-43 and hnRNP A1

TDP-43 ${ }^{1-274}$ : TDP-43 $3^{1-274}$ was overexpressed in BL21 (DE3) E. coli (TransGenBiotech, CD601-03) at $19^{\circ} \mathrm{C}$ for $16 \mathrm{~h}$ after induction by adding $50 \mu \mathrm{M}$ of IPTG. Briefly, cells were harvested by centrifugation at $4000 \mathrm{rpm}$ for $20 \mathrm{~min}$ at $4{ }^{\circ} \mathrm{C}$ and lysed with $50 \mathrm{~mL}$ of lysis buffer $(50 \mathrm{mM}$ Tris- $\mathrm{HCl}, 500 \mathrm{mM} \mathrm{NaCl}, \mathrm{pH} 8.0,10 \mathrm{mM}$ imidazole, $4 \mathrm{mM}$ 3-mercaptoethanol, $1 \mathrm{mM}$ PMSF, and $0.1 \mathrm{mg} / \mathrm{mL}$ RNase A). After the cell lysates were filtered with a $0.22 \mu \mathrm{m}$ filter, the protein was purified by Ni column (GE Healthcare, USA), eluted in an elution buffer $(50 \mathrm{mM}$ Tris- $\mathrm{HCl}, 500 \mathrm{mM} \mathrm{NaCl}, \mathrm{pH}$ 8.0, $250 \mathrm{mM}$ 
imidazole and $4 \mathrm{mM} \beta$-mercaptoethanol). The proteins were further purified by Superdex 200 16/600 column (GE Healthcare) in a buffer containing $50 \mathrm{mM}$ Tris- $\mathrm{HCl} \mathrm{pH} \mathrm{7.5,300} \mathrm{mM} \mathrm{NaCl}$ and $2 \mathrm{mM} \mathrm{DTT}$, and freshly frozen in liquid nitrogen and stored at $-80^{\circ} \mathrm{C}$.

TDP-43 ${ }^{274-414}$ : TDP-43 $3^{274-414}$ was expressed in BL21 (DE3) E. coli (TransGenBiotech, CD601-03) into inclusion bodies. Briefly, cells were harvested and lysed in a denatured lysis buffer (50 mM Tris$\mathrm{HCl}, \mathrm{pH} 8.0$ and $6 \mathrm{M}$ guanidine hydrochloride) at room temperature. Cell lysate was sonicated, followed by centrifugation at $14,000 \mathrm{rpm}$ for $1 \mathrm{~h}$ at $4{ }^{\circ} \mathrm{C}$. The protein was purified from the supernatant by using a $\mathrm{Ni}$ column with the elution buffer containing $50 \mathrm{mM}$ Tris- $\mathrm{HCl}$ at $\mathrm{pH} 8.0,6 \mathrm{M}$ guanidine hydrochloride and $50 \mathrm{mM}$ imidazole. After further purification by HPLC (Agilent) with an elution buffer containing $35 \%(\mathrm{v} / \mathrm{v})$ acetonitrile, TDP$43^{274-414}$ was freeze-dried by FreeZone Lyophilizers (Thermo Fisher) and stored at $-20^{\circ} \mathrm{C}$.

hnRNP A1: hnRNP A1 was expressed in BL21 (DE3) pLysS E. coli after adding $0.4 \mathrm{mM} \mathrm{IPTG}$ at $25^{\circ} \mathrm{C}$ overnight. Cells were harvested and lysed in a lysis buffer $(50 \mathrm{mM}$ Tris- $\mathrm{HCl}$ at $\mathrm{pH} 7.5,2 \mathrm{mM}$ DT, $1 \mathrm{mM}$ PMSF, $5 \%$ glycerin, and $0.1 \mathrm{mg} / \mathrm{mL}$ RNase A) at $4{ }^{\circ} \mathrm{C}$. The supernatant was loaded onto a $5 \mathrm{~mL}$ SP column by using a ÄKTA Purifier (GE Healthcare, USA). The proteins were eluted with a gradient mixing of buffer A $(50 \mathrm{mM}$ Tris- $\mathrm{HCl}$ pH 7.5, $2 \mathrm{mM}$ DTT and $5 \%$ glycerin) and buffer $B$ (buffer $A$ with $1 \mathrm{M} \mathrm{NaCl}$ ). hnRNP A1 protein was further purified by the Superdex $7516 / 600$ column (GE Healthcare) in a buffer containing $50 \mathrm{mM}$ Tris- $\mathrm{HCl} \mathrm{pH} \mathrm{7.5,500}$ $\mathrm{mM} \mathrm{NaCl}$ and $2 \mathrm{mM}$ DTT. Fractions containing hnRNP A1 monomers were collected and concentrated for the subsequent in vitro PARylation and phase separation assays.

MBP-WT and MBP-PBM ${ }^{\text {mut }}$ hnRNP A1: PBM $^{\text {mut }}$ hnRNP A1 protein was extremely insoluble when expressed in $E$. coli. and had to be purified with the MBP tag. The MBP-hnRNP A1 (WT or $\mathrm{PBM}^{\text {mut }}$ ) was expressed in E. coli Rosseta (DE3) competent cells with $0.5 \mathrm{mM}$ IPTG at $16^{\circ} \mathrm{C}$ overnight. Cells were harvested and lysed in a lysis buffer $(50 \mathrm{mM}$ Tris- $\mathrm{HCl}$ at $\mathrm{pH} 7.5,500 \mathrm{mM} \mathrm{NaCl}$, $4 \mathrm{mM} \beta$-mercaptoethanol, $1 \mathrm{mM}$ PMSF, $5 \%$ glycerin and $0.1 \mathrm{mg} /$ $\mathrm{mL}$ RNase A) at $4{ }^{\circ} \mathrm{C}$. The supernatant was loaded onto a $\mathrm{Ni}$ column by using a ÄKTA Purifier (GE Healthcare, USA). Proteins were eluted with a gradient mixing of buffer A $(50 \mathrm{mM}$ Tris- $\mathrm{HCl}$ $\mathrm{pH} 7.5,500 \mathrm{mM} \mathrm{NaCl}, 4 \mathrm{mM} \beta$-mercaptoethanol and $5 \%$ glycerin) and buffer B (buffer A with $500 \mathrm{mM}$ imidazole). The proteins were further purified using the Superdex $20016 / 600$ columns (GE Healthcare) in a buffer containing $50 \mathrm{mM}$ Tris- $\mathrm{HCl} \mathrm{pH} 7.5$, $500 \mathrm{mM} \mathrm{NaCl}$ and $2 \mathrm{mM}$ DTT. Fractions containing MBP-WT or MBP-PBM $^{\text {mut }}$ hnRNP A1 protein monomers were collected and concentrated for the subsequent in vitro phase separation and dot-blot binding assays.

All the purified proteins were confirmed by Coomassie brilliant blue staining and Western blotting. The RNase A is routinely applied during the process of RBPs protein purification in the laboratory. Furthermore, the agarose gel electrophoresis confirmed the absence of RNA contamination in the purified protein samples.

In vitro PARylation assay

The in vitro PARylation assay was performed according to the protocol adapted from. ${ }^{17}$ Briefly, substrate proteins were incubated with PARP1 (Sino Biological) in the presence of NAD in a reaction buffer $(50 \mathrm{mM}$ Tris- $\mathrm{HCl}, \mathrm{pH} 7.4,2 \mathrm{mM} \mathrm{MgCl}$ ) with or without $2.5 \mu \mathrm{g}$ of ssDNA (Sigma) at $37^{\circ} \mathrm{C}$ for $30 \mathrm{~min}$. The reactions were stopped by adding $20 \mu \mathrm{M}$ Olaparib and the products were examined by SDS-PAGE and Western blotting.

\section{PAR-binding dot-blot assay}

One microgram of purified proteins was diluted to $40 \mathrm{mM}$ Hepes $(\mathrm{PH} 7.4), 150 \mathrm{mM} \mathrm{NaCl}$ and $5 \%$ glycerol, and then blotted onto a $0.45 \mu \mathrm{m}$ nitrocellulose membrane. The membranes were left to dry for $30 \mathrm{~min}$ and then stained with Ponceau S for $10 \mathrm{~s}$. After the images were captured using an Amersham Imager 600 (GE Healthcare), the membranes were washed in PBST (0.05\% TWEEN 20 in PBS) for $30 \mathrm{~min}$ and then incubated in $1 \mathrm{~mL}$ of PBST containing $50 \mathrm{nM}$ PAR polymer for $1 \mathrm{~h}$ with rocking and rotation at room temperature. The membrane was washed in PBST and the immunoblotted with the primary and secondary antibodies and imaged similar to the procedure described in Western blotting above.

In vitro liquid-liquid phase separation (LLPS) assay

For the LLPS assay, purified hnRNP A1 or TDP-43 protein were mixed with PAR polymers (Trevigen, 4336-100-01) and $\mathrm{NaCl}$ at the indicated concentrations in a LLPS buffer $(50 \mathrm{mM}$ Tris- $\mathrm{HCl}, \mathrm{pH}$ 7.5, 10\% (w/v) PEG 3550 (Sigma) and 2 mM DTT) and incubated for $3 \mathrm{~min}$ at room temperature. For the co-LLPS, the purified hnRNP A1 and TDP-43 proteins were incubated in a co-LLPS buffer (50 mM Tris- $\mathrm{HCl}, \mathrm{pH} 7.5$, and $2 \mathrm{mM}$ DTT) with the indicated concentration of $\mathrm{NaCl}$. Finally, $5 \mu \mathrm{L}$ of each sample was pipetted onto a coverslip and imaged using a Leica microscope with differential interference contrast (DIC).

\section{Centrifugation-based phase separation assay}

The semi-quantitative, centrifugation-based phase separation assay was performed according to the protocol published in. ${ }^{43}$ Briefly, the mixture in the phase separation assay described above was centrifuged at $14,000 \times g$ for 2 min at room temperature. The pellet was then washed with the co-LLPS buffer $(50 \mathrm{mM}$ Tris- $\mathrm{HCl}$, $\mathrm{pH} 7.5,100 \mathrm{mM} \mathrm{NaCl}$ ) and re-suspend in the co-LLPS buffer. Next, an equal volume of the supernatant and the re-suspended pellet were separated by $12 \%$ Bis-Tris SDS-PAGE (Invitrogen) and the gel was stained with Coomassie blue. Finally, images were captured using an Amersham Imager 600 (GE Healthcare), whereas the densitometry of the bands was measured with the ImageQuant TL Software (GE Healthcare).

\section{Fluorophore-labeled hnRNP A1 and TDP-43}

Purified TDP- $43^{1-274}$ and hnRNP A1 proteins in storage buffer were desalted in a reaction buffer $(50 \mathrm{mM}$ Tris- $\mathrm{HCl}, \mathrm{pH} 7.5,500 \mathrm{mM} \mathrm{NaCl}$ and $4 \mathrm{mM}$ Tris (2-Carboxyethyl) Phosphine (TCEP) (Invitrogen, T2556) using a desalting column (GE Healthcare, USA) to remove DTT. The proteins were then incubated with a 5 -fold AlexaFluor555 C2-malemide (Invitrogen, A20346) for TDP-43 ${ }^{1-274}$ oran AlexaFluor-647 C2-malemide (Invitrogen, A20347) for hnRNPA1 at room temperature for $2 \mathrm{~h}$ to conjugate the maleimide derivative dye to a thiol group of thecysteine in the target proteins. The labeled proteins were further purified using the Superdex 200 10/300 columns (GE Healthcare, USA) in a buffer containing $50 \mathrm{mM}$ Tris $-\mathrm{HCl}, \mathrm{pH} 7.5,500 \mathrm{mM} \mathrm{NaCl}$, the unlabeled ones were mixed with $1 \%$ of the labeled proteins for the subsequent in vitro phase separation experiment and confocal imaging.

Fluorescence recovery after photobleaching (FRAP) assay

The PFRP assay was performed using the FRAP module of the Leica SP8 confocal microscopy system. In brief, the Alexa Fluor647-labeled hnRNPA1 was bleached using a 647-nm laser beam. Bleaching was focused on a circular region of interest (ROI) set to 2 $\mu \mathrm{m}$ in diameter. After photobleaching, time-lapse images were captured. For each indicated time point $(\mathrm{t})$, the fluorescence intensity within the bleached droplet was normalized to the fluorescence intensity of a nearby unbleached droplet. The normalized fluorescence intensity of pre-bleaching was set to $100 \%$ and the normalized fluorescence intensity at each time point $\left(I_{t}\right)$ was used to calculate the fluorescence recovery (FR) according to the following formula: $F R(t)=I_{t} / I_{\text {pre-bleaching. Image } J}$ was used for quantification and GraphPad Prism to plot and analyze the FRAP experiments. 
Lentivirus production and infection

293T cells were co-transfected with psPAX2, pMD2.G and pCDHCMV-MCS-EF1-Puro plasmids of Flag, TDP-43-Flag and hnRNP A1$\mathrm{HA}$ using the PolyJet ${ }^{\mathrm{TM}}$ reagent. The cell culture medium was collected and filtered through a $0.45 \mu \mathrm{m}$ syringe filter (Millipore, SLHV033RB) at $48 \mathrm{~h}$ after transfection. The lentivirus was then concentrated using the Lenti- $X^{\mathrm{TM}}$ Concentrator (Clontech, PT4421-2). The 20-fold concentrated medium containing hnRNP A1-expressing lentivirus or the 10-fold concentrated medium containing TDP-43-expressing lentivirus was used to infect NSC-34 cells in the subsequent experiments.

\section{Cell viability assay}

NSC-34 cells were seeded into 96-well plates (Corning) at the density of $1.28 \times 10^{4}$ cells/well and cultured in $100 \mu \mathrm{L}$ of culture medium containing the lentivirus particles for the expression of the proteins of interest. After $48 \mathrm{~h}$, the infection medium containing the lentivirus was removed and replaced with fresh medium. 48-72 $\mathrm{h}$ post infection, cell viability was examined using the Cell Counting Kit-8 (CCK-8) (Dojindo), according to the manufacturer's instructions. Briefly, $10 \mu \mathrm{L}$ of the CCK-8 solution were added to each well and incubated at $37^{\circ} \mathrm{C}$ for $2.5 \mathrm{~h}$. Finally, the absorbance at $450 \mathrm{~nm}$ was measured with a Synergy2 microplate reader (BioTek Instruments).

\section{TUNEL staining}

The TUNEL staining assay was performed using the TMR red in situ Cell Death Detection Kit (Sigma-Aldrich), according to the manufacturer's instructions. The transfected NSC-34 cells grown on the coverslips in a 24-well plate were fixed with $4 \%$ paraformaldehyde for $15 \mathrm{~min}$ and permeabilized with PBST $(1 \times$ PBS $+0.5 \%$ Triton $X-100$ ) for $10 \mathrm{~min}$ at room temperature, followed by incubation with $18 \mu \mathrm{L}$ of the labeling solution and $2 \mu \mathrm{L}$ of the enzyme solution at $37^{\circ} \mathrm{C}$ for $1 \mathrm{~h}$. Finally, cells were mounted on glass slides using the Vectashield Antifade Mounting Medium with DAPI (Vector Laboratories) and imaged with the Leica TCS SP8 confocal microscopy system.

\section{Drosophila strains}

The following strains were obtained from the Bloomington Drosophila Stock Center (BDSC): RNAi-Parp (\#57265), elavGS (\#43642), RNAi-mCherry (\#35785, a control for in vivo RNAi knockdown), UAS-LacZ (\#8529, a control for UAS transgene expression). The UAS-Parg fly strain was obtained from the Kyoto Stock Center (DGRC, \#109116). The transgenic fly strain of UASTDP-43 was generated by the ФC31 integrase-mediated, sitespecific integration into the fly genome, which allowed a uniform transgene expression across different lines. The attP landing site stock used in this study was UAS-phi2b2a;VK5 (75B1) and a transgenic pBID-UASC-Luciferase (UAS-Luc) fly strain was generated using the same method, the same landing site ${ }^{64}$ was used as a control in the this study. All flies were raised on a standard cornmeal media and maintained at $25^{\circ} \mathrm{C}$ and $60 \%$ relative humidity.

Climbing ability and Lifespan assays

For the climbing assays, 20 flies per vial, 5-8 vials per group were tested. All flies were transferred into an empty polystyrene vial, gently tapped down to the bottom and allowed 15 min time for flies to recover. The number of flies climbing over the height of $3 \mathrm{~cm}$ within 10 seconds was recorded. The test was repeated three times for each vial and 6 8 vials per group were tested.

For the lifespan experiments, 20 flies per vial, 7-9 vials per group were tested. Flies were transferred to the fresh fly food every 3 days. The flies lost prior to natural death because of escape or accidental death were excluded from the final analysis. The median lifespan was calculated as the mean of the medians of each vial in a group, the " $50 \%$ survival" shown on the survival curves is derived from the compilation of all vials of a group. For adult-onset, the neuronal expression of the RNAi transgenes was obtained using the elav-GS driver. ${ }^{65}$ Flies were raised at $25^{\circ} \mathrm{C}$ and $60 \%$ relative humidity on regular fly food supplemented with $80 \mu \mathrm{g} / \mathrm{mL}$ RU486 (TCl).

Statistical analysis

Unless otherwise noted, the statistical significance in this study is determined by the two-way ANOVA with Bonferroni's post-hoc test or the unpaired, two-tailed Student's $t$-test at ${ }^{*} p<0.05,{ }^{* *} p<$ 0.01 , and ${ }^{* * *} p<0.001$. The error bars represent the standard error of the mean (SEM).

\section{ACKNOWLEDGEMENTS}

We thank the BDSC and the Kyoto DGRC for providing fly strains, L. Pan for the pET32M.3C plasmid, B. Yang and X. Duan for help in in vitro PARylation assay, S. Qiu and S. Zhang for technical supports, and J. Yuan, A. Li and members of the Zhang lab, the Jiang lab, the Liu lab, and the Fang lab for helpful discussion and critical reading of the manuscript. This study was supported by grants from the National Key R\&D Program of China (No. 2016YFA0501902), the National Natural Science Foundation of China (No. 81671254 and No. 31471017) to Y.F., (No. 91853112 and No. 31470748) to C.L., (No. 21778063 and No. 91753114) to H.J., (No. 31500665 and No. 31671428) to Y.Z., and funding from the Shanghai Science and Technology Committee (No. 18ZR1448300) to A.D.

\section{AUTHOR CONTRIBUTIONS}

Y.D., C.L. and Y.F. conceived the research; Y.D., A.D., Y.Z., H.J., C.L. and Y.F. designed the experiments; Y.D., A.D., J.G., L.S., G.D., C.W., X.G., Z.M., L.S., B.Q. and K.T. performed the experiments; Y.D., A.D., G.D., C.W., Z.M., X.D. and H.J. contributed important new reagents; Y.D., A.D., J.G., G.D., C.W., Z.M., L.S., K.Z. and Y.Z. analyzed the data; Y.D., A.D., J.G., L.S., C.L. and Y.F. interpreted and discussed the results; Y.D., A.D., J.G., G.D. and Y.F. prepared the figures; and Y.D., A.D., G.D., C.L. and Y.F. wrote the paper. All authors read and approved the final manuscript.

\section{ADDITIONAL INFORMATION}

Supplementary information accompanies this paper at https://doi.org/10.1038/ s41422-019-0141-z.

Competing interests: Y.F. and Y.D. have filed an international patent via the PCT entitled "Application of PARP1 Inhibitors for Treating Patients with Amyotrophic Lateral Sclerosis". The remaining authors declare no conflict of interests.

\section{REFERENCES}

1. Anko, M. L. \& Neugebauer, K. M. RNA-protein interactions in vivo: global gets specific. Trends Biochem. Sci. 37, 255-262 (2012).

2. Lunde, B. M., Moore, C. \& Varani, G. RNA-binding proteins: modular design for efficient function. Nat. Rev. Mol. Cell Biol. 8, 479-490 (2007).

3. Muller-McNicoll, M. \& Neugebauer, K. M. How cells get the message: dynamic assembly and function of mRNA-protein complexes. Nat. Rev. Genet. 14, 275-287 (2013).

4. Lin, Y., Protter, D. S., Rosen, M. K. \& Parker, R. Formation and maturation of phaseseparated liquid droplets by RNA-binding proteins. Mol. Cell 60, 208-219 (2015).

5. Van Damme, P., Robberecht, W. \& Van Den Bosch, L. Modelling amyotrophic lateral sclerosis: progress and possibilities. Dis. Model Mech. 10, 537-549 (2017).

6. Brady, O. A., Meng, P., Zheng, Y., Mao, Y. \& Hu, F. Regulation of TDP-43 aggregation by phosphorylation and p62/SQSTM1. J. Neurochem. 116, 248-259 (2011).

7. Cohen, T. J. et al. An acetylation switch controls TDP-43 function and aggregation propensity. Nat. Commun. 6, 5845 (2015).

8. Dammer, E. B. et al. Coaggregation of RNA-binding proteins in a model of TDP-43 proteinopathy with selective RGG motif methylation and a role for RRM1 ubiquitination. PLoS One 7, e38658 (2012).

9. Li, W. et al. Heat Shock-induced phosphorylation of TAR DNA-binding Protein 43 (TDP-43) by MAPK/ERK kinase regulates TDP-43 function. J. Biol. Chem. 292, 5089-5100 (2017).

10. Hofweber, M. et al. Phase Separation of FUS Is Suppressed by its nuclear import receptor and arginine methylation. Cell 173, 706-719 e713 (2018).

11. Qamar, S. et al. FUS Phase separation is modulated by a molecular chaperone and methylation of arginine cation-pi interactions. Cell 173, 720-734 e715 (2018). 
12. Ryan, V. H. et al. Mechanistic view of hnRNPA2 low-complexity domain structure, interactions, and phase separation altered by mutation and arginine methylation. Mol. Cell 69, 465-479 e467 (2018).

13. Luo, F. et al. Atomic structures of fus Ic domain segments reveal bases for reversible amyloid fibril formation. Nat. Struct. Mol. Biol. 25, 341-346 (2018).

14. Martello, R. et al. Proteome-wide identification of the endogenous ADPribosylome of mammalian cells and tissue. Nat. Commun. 7, 12917 (2016).

15. Zhang, Y., Wang, J., Ding, M. \& Yu, Y. Site-specific characterization of the Asp- and Glu-ADP-ribosylated proteome. Nat. Methods 10, 981-984 (2013).

16. Niere, $M$. et al. ADP-ribosylhydrolase 3 (ARH3), not poly(ADP-ribose) glycohydrolase (PARG) isoforms, is responsible for degradation of mitochondrial matrixassociated poly(ADP-ribose). J. Biol. Chem. 287, 16088-16102 (2012).

17. Slade, D. et al. The structure and catalytic mechanism of a poly(ADP-ribose) glycohydrolase. Nature 477, 616-620 (2011).

18. Ahel, I. et al. Poly(ADP-ribose)-binding zinc finger motifs in DNA repair/checkpoint proteins. Nature 451, 81-85 (2008).

19. Andrabi, S. A. et al. Poly(ADP-ribose) (PAR) polymer is a death signal. Proc. Natl. Acad. Sci. USA 103, 18308-18313 (2006).

20. Frizzell, K. M. et al. Global analysis of transcriptional regulation by poly(ADPribose) polymerase-1 and poly(ADP-ribose) glycohydrolase in MCF-7 human breast cancer cells. J. Biol. Chem. 284, 33926-33938 (2009).

21. Singh, H. R. et al. A Poly-ADP-Ribose trigger releases the auto-inhibition of a chromatin remodeling oncogene. Mol. Cell 68, 860-871 e867 (2017).

22. Deng, W. PARylation: strengthening the connection between cancer and pluripotency. Cell. Stem. Cell. 5, 349-350 (2009).

23. Hanai, S. et al. Loss of poly(ADP-ribose) glycohydrolase causes progressive neurodegeneration in Drosophila melanogaster. Proc. Natl. Acad. Sci. USA 101, 82-86 (2004).

24. Martire, S., Mosca, L. \& d'Erme, M. PARP-1 involvement in neurodegeneration: a focus on Alzheimer's and Parkinson's diseases. Mech. Ageing Dev. 146-148, 53-64 (2015).

25. Brochier, C., Jones, J. I., Willis, D. E. \& Langley, B. Poly(ADP-ribose) polymerase 1 is a novel target to promote axonal regeneration. Proc. Natl. Acad. Sci. USA 112, 15220-15225 (2015).

26. Leung, A. K. et al. Poly(ADP-ribose) regulates stress responses and microRNA activity in the cytoplasm. Mol. Cell 42, 489-499 (2011).

27. Kim, $\mathrm{H}$. J. et al. Mutations in prion-like domains in hnRNPA2B1 and hnRNPA1 cause multisystem proteinopathy and ALS. Nature 495, 467-473 (2013).

28. Neumann, M. et al. Ubiquitinated TDP-43 in frontotemporal lobar degeneration and amyotrophic lateral sclerosis. Science 314, 130-133 (2006).

29. Buratti, E. et al. TDP-43 binds heterogeneous nuclear ribonucleoprotein A/B through its $C$-terminal tail: an important region for the inhibition of cystic fibrosis transmembrane conductance regulator exon 9 splicing. J. Biol. Chem. 280, 37572-37584 (2005).

30. Lee, E. B., Lee, V. M. \& Trojanowski, J. Q. Gains or losses: molecular mechanisms of TDP43-mediated neurodegeneration. Nat. Rev. Neurosci. 13, 38-50 (2012).

31. Geuens, T., Bouhy, D. \& Timmerman, V. The hnRNP family: insights into their role in health and disease. Hum. Genet. 135, 851-867 (2016).

32. Mohagheghi, F. et al. TDP-43 functions within a network of hnRNP proteins to inhibit the production of a truncated human SORT1 receptor. Hum. Mol. Genet. 25, 534-545 (2016).

33. Gagne, J. P., Hunter, J. M., Labrecque, B., Chabot, B. \& Poirier, G. G. A proteomic approach to the identification of heterogeneous nuclear ribonucleoproteins as a new family of poly(ADP-ribose)-binding proteins. Biochem. J. 371, 331-340 (2003).

34. Gagne, J. P. et al. Proteome-wide identification of poly(ADP-ribose) binding proteins and poly(ADP-ribose)-associated protein complexes. Nucleic Acids Res. 36, 6959-6976 (2008)

35. Fong, P. C. et al. Inhibition of poly(ADP-ribose) polymerase in tumors from BRCA mutation carriers. N. Engl. J. Med. 361, 123-134 (2009).

36. Gottschald, O. R. et al. TIAR and TIA-1 mRNA-binding proteins co-aggregate under conditions of rapid oxygen decline and extreme hypoxia and suppress the HIF-1alpha pathway. J. Mol. Cell Biol. 2, 345-356 (2010).

37. Kim, M. Y., Mauro, S., Gevry, N., Lis, J. T. \& Kraus, W. L. NAD +-dependent modulation of chromatin structure and transcription by nucleosome binding properties of PARP-1. Cell 119, 803-814 (2004).

38. Poirier, G. G., de Murcia, G., Jongstra-Bilen, J., Niedergang, C. \& Mandel, P. Poly (ADP-ribosyl)ation of polynucleosomes causes relaxation of chromatin structure. Proc. Natl. Acad. Sci. USA 79, 3423-3427 (1982).

39. He, Y. \& Smith, R. Nuclear functions of heterogeneous nuclear ribonucleoproteins A/B. Cell. Mol. Life Sci. 66, 1239-1256 (2009).
40. Siomi, H. A nuclear localization domain in the hnRNP A1 protein. J. Cell. Biol. 129, 551-560 (1995).

41. Nichols, R. C. et al. The RGG domain in hnRNP A2 affects subcellular localization. Exp. Cell Res. 256, 522-532 (2000).

42. Naruse, H. et al. Molecular epidemiological study of familial amyotrophic lateral sclerosis in Japanese population by whole-exome sequencing and identification of novel HNRNPA1 mutation. Neurobiol. Aging 61, 255 e259-255 e216 (2018).

43. Zeng, M. et al. Phase transition in postsynaptic densities underlies formation of synaptic complexes and synaptic plasticity. Cell 166, 1163-1175 e1112 (2016).

44. Dreyfuss, G., Matunis, M. J., Pinol-Roma, S. \& Burd, C. G. hnRNP proteins and the biogenesis of mRNA. Annu. Rev. Biochem. 62, 289-321 (1993).

45. D'Ambrogio, A. et al. Functional mapping of the interaction between TDP-43 and hnRNP A2 in vivo. Nucleic Acids Res. 37, 4116-4126 (2009).

46. Chang, P., Jacobson, M. K. \& Mitchison, T. J. Poly(ADP-ribose) is required for spindle assembly and structure. Nature 432, 645-649 (2004).

47. Kotova, E., Jarnik, M. \& Tulin, A. V. Poly (ADP-ribose) polymerase 1 is required for protein localization to Cajal body. PLoS Genet. 5, e1000387 (2009).

48. Ahel, D. et al. Poly(ADP-ribose)-dependent regulation of DNA repair by the chromatin remodeling enzyme ALC1. Science 325, 1240-1243 (2009).

49. Anderson, P. \& Kedersha, N. RNA granules: post-transcriptional and epigenetic modulators of gene expression. Nat. Rev. Mol. Cell Biol. 10, 430-436 (2009).

50. Buchan, J. R. \& Parker, R. Eukaryotic stress granules: the ins and outs of translation. Mol. Cell 36, 932-941 (2009).

51. Zhang, K. et al. Stress granule assembly disrupts nucleocytoplasmic transport. Cell 173, 958-971 e917 (2018).

52. McGurk, L. et al. Poly(ADP-Ribose) prevents pathological phase separation of tdp43 by promoting liquid demixing and stress granule localization. Mol. Cell 71, 703-717 e709 (2018)

53. Shieh, W. M. et al. Poly(ADP-ribose) polymerase null mouse cells synthesize ADPribose polymers. J. Biol. Chem. 273, 30069-30072 (1998).

54. lijima, M., Suzuki, M., Tanabe, A., Nishimura, A. \& Yamada, M. Two motifs essential for nuclear import of the hnRNP A1 nucleocytoplasmic shuttling sequence M9 core. FEBS Lett. 580, 1365-1370 (2006).

55. Suzuki, H., Shibagaki, Y., Hattori, S. \& Matsuoka, M. Nuclear TDP-43 causes neuronal toxicity by escaping from the inhibitory regulation by hnRNPs. Hum. Mol. Genet. 24, 1513-1527 (2015).

56. Davar, D., Beumer, J. H., Hamieh, L. \& Tawbi, H. Role of PARP inhibitors in cancer biology and therapy. Curr. Med. Chem. 19, 3907-3921 (2012).

57. Bai, P. Biology of poly(ADP-Ribose) polymerases: the factotums of cell maintenance. Mol. Cell 58, 947-958 (2015).

58. Cardinale, A., Paldino, E., Giampa, C., Bernardi, G. \& Fusco, F. R. PARP-1 inhibition is neuroprotective in the R6/2 mouse model of huntington's disease. PLoS One 10, e0134482 (2015).

59. Egi, Y. et al. Neuroprotective effects of a novel water-soluble poly(ADP-ribose) polymerase-1 inhibitor, MP-124, in in vitro and in vivo models of cerebral ischemia. Brain Res. 1389, 169-176 (2011).

60. Teng, F. et al. Neuroprotective effects of poly(ADP-ribose) polymerase Inhibitor olaparib in transient cerebral ischemia. Neurochem. Res. 41, 1516-1526 (2016).

61. Kam, T.-I. et al. Poly(ADP-ribose) drives pathologic a-synuclein neurodegeneration in Parkinson's disease. Science 362, eaat8407 (2018).

62. Jiang, L. L. et al. Structural transformation of the amyloidogenic core region of TDP-43 protein initiates its aggregation and cytoplasmic inclusion. J. Biol. Chem. 288, 19614-19624 (2013).

63. Chen, $\mathrm{Y}$. et al. Activity-induced Nr4a1 regulates spine density and distribution pattern of excitatory synapses in pyramidal neurons. Neuron 83, 431-443 (2014).

64. Cao, $X$. et al. In vivo imaging reveals mitophagy independence in the maintenance of axonal mitochondria during normal aging. Aging Cell. 16, 1180-1190 (2017).

65. Osterwalder, T., Yoon, K. S., White, B. H. \& Keshishian, H. A conditional tissuespecific transgene expression system using inducible GAL4. Proc. Natl. Acad. Sci. USA 98, 12596-12601 (2001).

66. Elden, A. C. et al. Ataxin-2 intermediate-length polyglutamine expansions are associated with increased risk for ALS. Nature 466, 1069-1075 (2010).

67. Ayala, Y. M. et al. TDP-43 regulates its mRNA levels through a negative feedback loop. EMBO J. 30, 277-288 (2011).

68. Polymenidou, M. et al. Long pre-mRNA depletion and RNA missplicing contribute to neuronal vulnerability from loss of TDP-43. Nat. Neurosci. 14, 459-468 (2011). 